\title{
Decreased gut microbiota diversity, delayed Bacteroidetes colonisation and reduced Th1 responses in infants delivered by Caesarean section
}

Hedvig E Jakobsson, Thomas R Abrahamsson, Maria C Jenmalm, Keith Harris, Christopher

Quince, Cecilia Jernberg, Bengt Björkstén, Lars Engstrand and Anders F Andersson

\author{
Linköping University Post Print
}

\section{Tweet}

N.B.: When citing this work, cite the original article.

Original Publication:

Hedvig E Jakobsson, Thomas R Abrahamsson, Maria C Jenmalm, Keith Harris, Christopher Quince, Cecilia Jernberg, Bengt Björkstén, Lars Engstrand and Anders F Andersson, Decreased gut microbiota diversity, delayed Bacteroidetes colonisation and reduced Th1 responses in infants delivered by Caesarean section, 2013, Gut.

http://dx.doi.org/10.1136/gutjnl-2012-303249

Copyright: BMJ Publishing Group http://group.bmj.com/

Postprint available at: Linköping University Electronic Press http://urn.kb.se/resolve?urn=urn:nbn:se:liu:diva-102209 
1 Decreased gut microbiota diversity, delayed Bacteroidetes colonization, and reduced

\section{Th1 responses in infants delivered by Caesarean section}

3 Running title: Development of the intestinal microbiota

4

5 Hedvig E Jakobsson ${ }^{1,2}$, Thomas R Abrahamsson ${ }^{3}$, Maria C Jenmalm ${ }^{3,4}$, Keith Harris ${ }^{5}$,

6 Christopher Quince ${ }^{5}$, Cecilia Jernberg ${ }^{1}$, Bengt Björkstén ${ }^{6,7}$, Lars Engstrand ${ }^{2 *}$, and Anders F

7 Andersson $^{8 *}$

$8{ }^{1}$ Department of Preparedness, Swedish Institute for Communicable Disease Control, Solna,

9 Sweden, ${ }^{2}$ Department of Microbiology, Tumor and Cell Biology, Karolinska Institutet,

10 Stockholm, Sweden, ${ }^{3}$ Department of Clinical and Experimental Medicine, Division of

11 Pediatrics, Linköping University, Sweden, ${ }^{4}$ Department of Clinical and Experimental

12 Medicine, Division of Inflammation Medicine, Linköping University, Sweden, ${ }^{5}$ School of

13 Engineering, University of Glasgow, Glasgow, United Kingdom, ${ }^{6}$ Institute of Environmental

14 Medicine, Karolinska Institutet, Stockholm, ${ }^{7}$ School of Health and Medical Sciences, Örebro

15 University, ${ }^{8}$ Science for Life laboratory, KTH Royal Institute of Technology, Stockholm,

16 Sweden.

$17 *$ Corresponding authors.

18 Anders F Andersson: KTH Royal Institute of Technology, Science for Life Laboratory,

19 School of Biotechnology, Division of Gene Technology, PO Box 1031, SE-171 21 Solna,

20 Sweden. Phone: +46-8-52481414. Email: anders.andersson@scilifelab.se

21 Lars Engstrand: Department of Microbiology, Tumor and Cell Biology, Karolinska Institutet,

22 SE-171 82 Stockholm, Sweden. Phone: +46-8-524 845 97. Email: lars.engstrand@ki.se

23 Keywords: chemokines/infant gut/intestinal bacteria/intestinal microbiology/molecular

24 biology

25 Word count: 3506 
1

2

3

4

5

6

7

8

9

10

11

12

13

14

15

16

17

18

19

20

21

22

23

24

25

26

27

28

29

30

31

32

33

34

35

36

37

38

39

40

41

42

43

44

45

46

47

48

49

50

51

52

53

54

55

56

57

58

59

60
26 Abbreviations: OTU, operational taxonomic unit; rRNA, ribosomal RNA; VD, vaginal

27 delivered; CS, caesarian section. 
28 The Corresponding Author has the right to grant on behalf of all authors and does grant on

29 behalf of all authors, an exclusive licence (or non exclusive for government employees) on a

30 worldwide basis to the BMJ Publishing Group Ltd and its Licensees to permit this article (if

31 accepted) to be published in Gut editions and any other BMJPGL products to exploit all

32 subsidiary rights, as set out in our licence.

33

34

35

36

37

38

39

40

41

42

43

44

45

46

47

48

49

50

51

52 
1

2

3

4

5

6

7

8

9

10

11

12

13

14

15

16

17

18

19

20

21

22

23

24

25

26

27

28

29

30

31

32

33

34

35

36

37

38

39

40

41

42

43

44

45

46

47

48

49

50

51

52

53

54

55

56

57

58

59

60

\section{AUTHOR CONTRIBUTIONS}

54 Conception and design: HEJ, TRA, MCJ, BB, CJ, LE, AFA

55 Analysis and interpretation of data: HEJ, TRA, MCJ, KH, CQ, AFA

56 Drafting the article: HEJ, TRA, MCJ, BB, AFA

57 Final approval of submitted version: HEJ, TRA, MCJ, KH, CQ, CJ, BB, LE, AFA

58

59

60

61

62

63

64

65

66

67

68

69

70

71

72

73

74

75

76

77

3

http://mc.manuscriptcentral.com/gut 


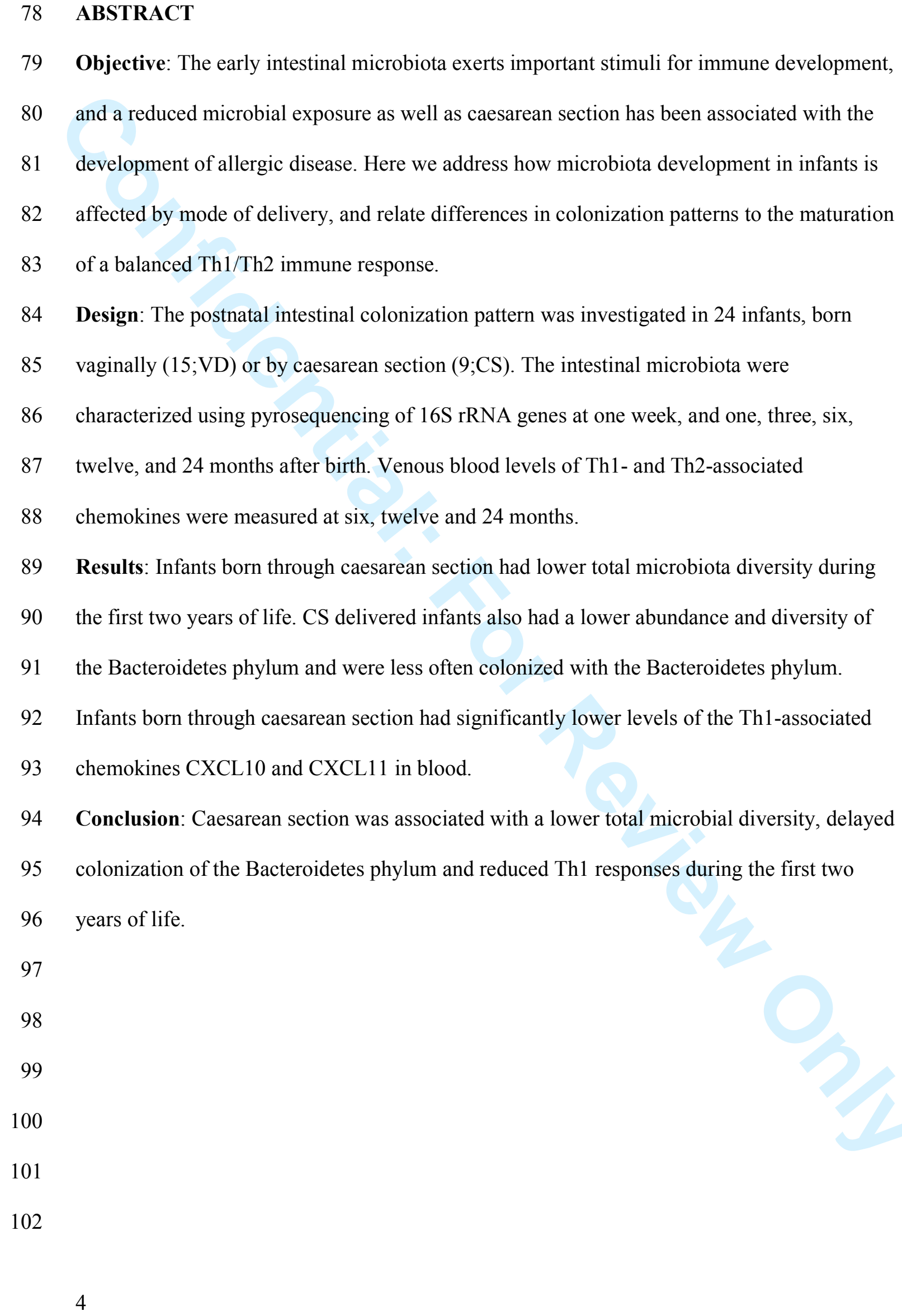

79 Objective: The early intestinal microbiota exerts important stimuli for immune development, 80 and a reduced microbial exposure as well as caesarean section has been associated with the 81 development of allergic disease. Here we address how microbiota development in infants is 82 affected by mode of delivery, and relate differences in colonization patterns to the maturation 83 of a balanced Th1/Th2 immune response.

84 Design: The postnatal intestinal colonization pattern was investigated in 24 infants, born 85 vaginally $(15 ; \mathrm{VD})$ or by caesarean section $(9 ; \mathrm{CS})$. The intestinal microbiota were 86 characterized using pyrosequencing of $16 \mathrm{~S}$ rRNA genes at one week, and one, three, six, 87 twelve, and 24 months after birth. Venous blood levels of Th1- and Th2-associated 88 chemokines were measured at six, twelve and 24 months.

89 Results: Infants born through caesarean section had lower total microbiota diversity during 90 the first two years of life. CS delivered infants also had a lower abundance and diversity of 91 the Bacteroidetes phylum and were less often colonized with the Bacteroidetes phylum.

92 Infants born through caesarean section had significantly lower levels of the Th1-associated 93 chemokines CXCL10 and CXCL11 in blood.

94 Conclusion: Caesarean section was associated with a lower total microbial diversity, delayed 95 colonization of the Bacteroidetes phylum and reduced Th1 responses during the first two 96 years of life. 
103

104 What is already known about this subject:

105 immune regulation. observed in several studies.

What are the new findings: years of life. two years of life. chemokines during infancy.
- The infant gut microbiota diversity increases during the first years of life.

- The microbiota composition differs between infants born by caesarean section or vaginal delivery with a delayed colonization of the genus Bacteroides.

- Bacterial colonization is necessary for the development of the immune system and

- An association between CS delivery and the development of allergic disease has been

- The total microbiota diversity is lower in CS than VD infants through the first two

- The diversity of the Bacteroidetes phylum is lower in CS born infants during the first

- Vaginal delivery is associated with increased circulating levels of Th1-associated

How might it impact on clinical practice in the foreseeable future?

- Deeper knowledge of the impact of delivery mode on microbiota composition and immune regulation may lead to improved allergy preventive strategies. 


\section{Introduction}

130 The gastrointestinal tract of the newborn infant is considered to be sterile. Bacteria from the

131 environment, mainly from the mother, colonize the infant gut immediately following birth.

132 Dominant members of anaerobic Firmicutes and Bacteroidetes do not appear to grow outside

133 the gut and hence need to be transmitted between human hosts [1]. To what extent the

134 transmission occurs from mother to offspring is not clear, but differences in microbiota

135 composition depending on delivery mode indicate a mother-child transmission during vaginal

136 delivery. A recent study based on pyrosequencing of 16S rRNA genes demonstrated that the

137 microbiota of vaginally delivered (VD) neonates ( $<24$ hours post delivery) resembled the

138 vaginal microbiota of their own mother and was similar across multiple body habitats (skin,

139 oral, nasopharynx and feces), while in neonates born by caesarean section (CS), it resembled

140 the mother's skin microbiota [2]. While this study provided evidence that microbiota from the

141 birth channel is transferred from mother to child, providing an inoculum for the initial

142 microbiota, it remains to be shown that specific gut microbes are successfully transmitted

143 during vaginal delivery.

144

145 The incidence of caesarean delivery has increased from $5 \%$ in the 1970 s to more than $60 \%$ in

146 some hospitals in China according to recent reports [3]. The early colonization pattern differs

147 between vaginally delivered infants and those delivered by caesarean section, including a

148 delayed colonization of e.g. Bacteroides and Bifidobacterium spp. in CS infants [4, 5]. The

149 influence on delivery mode on gut microbiota development has not previously been

150 longitudinally characterized using powerful cultivation-independent microbiologic methods,

151 however. The intestinal microbiota is important for the development of the immune system

152 and immunological tolerance [6]. Differences in the postnatal microbial colonization may

153 explain the higher incidence of immune mediated diseases such as allergy in children born by 
154 CS as compared to those born vaginally [7, 8]. Indeed, allergic disease has been associated

155 with low prevalence of Bacteroides and Bifidobacterium $[9,10]$, and a low intestinal

156 microbiota biodiversity in early infancy appears to have an impact on the development of

157 allergic disease later in life $[11,12,13]$. A failure of Th2-silencing during maturation of the

158 immune system may underlie development of Th2-mediated allergic disease [14].

159 Appropriate microbial stimulation may be required to avoid this pathophysiological process,

160 as early differences in the gut microbiota may shape later immune development $[6,15]$. The

161 influence of CS on immune development is largely unknown, however [16]. The aim of the

162 present study was to monitor the development of the infant intestinal microbiota in babies

163 born vaginally and through caesarian section, and to relate the findings to the maternal

164 microbiota and to Th1- and Th2-associated chemokine levels during infancy.

166 MATERIALS AND METHODS

167

168 Ethics

169 The human ethic committee at Linköping University, Linköping, Sweden, approved the

170 study. Informed consent was obtained from both parents before inclusion.

172 Subjects and sample collection

173 The study group comprised 24 healthy women and their infants. Nine of the infants were born

174 by caesarean delivery (CS) and 15 by vaginal delivery (VD) (c.f. Supplementary Table 1

175 regarding, sex, delivery, birth weight, the use of antimicrobials, and length of breast-feeding

176 for the different infants). Seven out of nine mothers, who gave birth through CS were given

177 antibiotics prophylactically during the surgery (Supplementary Table 2). This was done after

178 the delivery, however, and thus the infants were not exposed to antibiotics via the placenta. 
179 No children were treated with antibiotics during the neonatal period. Twenty of the infants

$180(83 \%)$ were partly breast fed until at least six months of age. The women and children

181 included in this study were part of a larger study assessing the prevention of allergic disease

182 by probiotics [17] (ClinicalTrials.gov ID NCT01285830) and they all received placebo. Stool

183 samples were collected from the mothers one week after delivery and from the children at

184 one week, one, three, six, twelve, and 24 months. The fecal samples were immediately frozen

185 at $-20^{\circ} \mathrm{C}$ following collections and later stored at $-70^{\circ} \mathrm{C}$. Samples were collected in $2002-$

1862005 and stored in $-70^{\circ} \mathrm{C}$ until DNA extractions were conducted in 2009 . No systematic

187 differences in storage times existed between the VD and CS samples (Mean months: VD:

$18880 \pm 7, \mathrm{CS}: 78 \pm 4$, Student's t-test, $\mathrm{P}=0.42$ ).

190 DNA extraction and 16S rRNA gene amplification

191 The DNA extraction and 16S rRNA gene amplification were performed as described

192 previously [18] with the following modifications; the primer pair used, targeting the variable

193 regions 3 and 4 of the 16S rRNA gene, was 341f 5'CCTACGGGNGGCWGCAG with

194 adaptor B and 805r 5'GACTACHVGGGTATCTAATCC with adaptor A and a sample-

195 specific barcode sequence consisting of five nucleotides [19]. The barcodes contained no

196 homopolymers and a pair of barcodes differed in at least two positions. The $341 \mathrm{f}-805 \mathrm{r}$ primer

197 pair was shown to be the least biased among 512 primer pairs evaluated in silico for bacterial

198 amplification and was experimentally shown to give a taxonomic composition similar to

199 shotgun metagenomics [20]. The primer pair has good coverage of bacterial groups typically

200 found in the human lower intestine. For phylum Bacteroidetes, 121,862 of 132,120, for

201 phylum Firmicutes 376,912 of 406,649, for phylum Proteobacteria 336,471 of 368,375 and

202 for family Bifidobacteriaceae 1112 of the 1239 sequences are matched, when considering

203 Ribosomal Database Project (RDP) sequences that span the region. A negative PCR reaction 
204 without template was also included for all primer pairs in each run. The PCR-products with

205 approximate lengths of 450 bp were purified with AMPure beads (Becton Dickinson,

206 Franklin Lakes, NJ, USA) using a Magnet Particle Separator (Invitrogen, Carlsbad, Calif.).

207 The concentrations of the purified products were measured by Qubit fluorometer (Invitrogen,

208 CA), the quality was assessed on a Bioanalyzer 2100 (Agilent, Santa Clara, CA, USA), and

209 the samples were amplified in PCR-mixture-in-oil emulsions and sequenced from the 805r

210 primer on different lanes of a 2-lane PicoTiterPlate on a Genome Sequencer FLX system

211 (Roche, Basel, Switzerland) at the Swedish Institute for Infectious Disease Control (Solna,

212 Sweden). Sequence processing was carried out with the AmpliconNoise software package

213 [21] correcting for errors introduced in the PCR and pyrosequencing as well as removing

214 chimeric sequences. Also, reads lacking a correct primer and/or having less than 360

215 successful pyrosequencing flows were excluded [21]. Denoised sequences were trimmed to

216198 bp after primer and barcode removal and clustered by complete linkage clustering into

217 Operational Taxonomic Units (OTUs) at the 97\% similarity level using AmpliconNoise.

218

219 Taxonomic classification

220 Each denoised sequence, as well as the most abundant sequence for each OTU, was BLAST

221 searched with default parameters against a local BLAST database comprising 836,814 near

222 full-length bacterial 16S rRNA gene sequences from the Ribosomal Database Project (RDP)

223 v. 10.10 [22]. The sequences inherited the taxonomic annotation (down to genus level) of the

224 best scoring RDP hit fulfilling the criteria of $\geq 95 \%$ identity over an alignment of length $\geq$

$225180 \mathrm{bp}$. If no such hit was found the sequence was classified as "no match". If multiple best

226 hits (of same score) were found, the taxonomy was set to the most-detailed level of taxonomy

227 shared by the best hits. The majority of reads had an RDP relative within $95 \%$ sequence

228 similarity and were hence of bacterial origin. After removal of pyrosequencing noise and 
229 chimeric sequences using the AmpliconNoise package [21], 357,685 high quality, typically

230198 bp long, sequence reads remained, with 828 to 4395 reads per sample $($ mean $=2129)$.

231 These corresponded to 3048 unique sequences and 1818 OTUs, clustered at 97\% similarity

232 level using complete linkage clustering.

233

234 Sample clustering

235 The online version of Fast Unifrac (http://bmf2.colorado.edu/fastunifrac/) [23] was used to

236 calculate weighted sample distances by mapping our OTU sequences with BLAST onto the

237 Greengenes reference sequences (downloaded from the Fast Unifrac web page, May 2009)

238 and using the corresponding Greengenes tree. A Principal Coordinates Analysis (PCoA) plot

239 based on all pair-wise sample distance was created on the Fast Unifrac web page. Our OTU

240 sequences were mapped onto 154 Greengenes sequences.

242 Statistical testing for over or under-representation of bacterial lineages

243 Statistical tests of over or under-representation of bacterial lineages among sample groups

244 were made at the phylum and genus levels using Wilcoxon rank-sum test. To correct for

245 multiple testing, the P-values were converted to False Discovery Rate values (Q-values).

247 Diversity estimations

248 Shannon diversity index was calculated as $H=-\Sigma \log \left(\mathrm{p}_{i}\right) \mathrm{p}_{i}$, where $\mathrm{p}_{i}$ denotes the relative

249 frequency of OTU $i$ [24], Pielou's eveness index as $-\Sigma \log \left(\mathrm{p}_{i}\right) \mathrm{p}_{i} / \log \left(S_{o b s}\right)$, where $S_{\text {obs }}$ denotes

250 the number of observed OTUs in the sample [25], and Chaol richness estimate as $S_{o b s}+\mathrm{n}_{l}\left(\mathrm{n}_{1-}\right.$

$2511) / \mathrm{n}_{2}\left(\mathrm{n}_{2}-1\right)$, where $\mathrm{n}_{1}$ and $\mathrm{n}_{2}$ are the number of observed singleton and doubleton OTUs,

252 respectively [26]. Since these parameters are influenced by sequencing depth and the

253 sequencing depth differed between samples, we subsampled (with replacement) 1400 reads 
254 from each sample, counted the occurrences of the corresponding OTUs, and performed the

255 diversity calculations on these counts. This was repeated ten times and averages of the

256 diversity parameters calculated and used for further analysis. Four (out of 168) samples had

257 fewer than 1400 reads and were excluded from this part of the analysis (three VD infants; one

258 at one week, one at three months and one at twelve months, and one CS infant at one month).

259 Diversity calculations and statistics were done with the $R$ software (http://www.r-project.org/)

260 and the $R$ package vegan (http://cran.r-project.org/web/packages/vegan/). Repeated measures

261 ANOVA were employed, using the Shannon diversity index at the different time points (one

262 week, one, three, six, twelve and 24 months) as the repeated measures.

263

264 Statistical testing of mother-child overlap in sequence types

265 For each time point and for each infant we calculated the number of specific sequences

266 (sequence types) shared with its mother/number of sequence types observed in the infant

$267\left(\mathrm{R}_{\mathrm{own}}\right)$. Likewise we calculated the average number of sequence types shared with other

268 mothers/number of sequence types observed in the infant $\left(\mathrm{R}_{\mathrm{other}}\right)$. We then compared the

$269 \mathrm{R}_{\text {own }}$ and $\mathrm{R}_{\text {other }}$ values pairwise for all infants within each group (VD or CS) with the

270 Wilcoxon signed rank test.

271

272 Chemokine analyses in venous blood and association with mode of delivery

273 Venous blood was collected at six $(n=24)$, twelve $(n=24)$ and 24 months $(n=24)$ and stored as 274 plasma or serum in $-20^{\circ} \mathrm{C}$ pending analysis. The Th1-associated chemokines CXCL10, and

275 CXCL11 and the Th2-associated chemokines CCL17 and CCL22 were analyzed with an in-

276 house multiplexed Luminex assay [27, 28]. The limit of detection was $6 \mathrm{pg} / \mathrm{ml}$ for CXCL10

277 and CXCL11 and $2 \mathrm{pg} / \mathrm{ml}$ for CCL17 and for CCL22. All samples were analysed in

278 duplicates and the sample was re-analysed if the coefficient of variance $(\mathrm{CV})$ was $>15 \%$. The 
279 chemokine levels were compared between infants being born vaginally or by caesarean

280 section by repeated measures ANOVA using log transformed chemokine levels at the

281 different time points (six, twelve and 24 months) as the repeated measures.

282

283 RESULTS

284

285 Microbiota succession in infants

286 At the phylum level, the microbiota developed in a similar fashion in infants delivered

287 vaginally and by CS, with a gradual decline in Proteobacteria from one week to 24 months, a

288 peak of Actinobacteria at three months, an expansion of Firmicutes from three months and

289 onward, and the emergence of Verrucomicrobia at around six months of age (Figure 1,

290 Supplementary Table 3). However, a notable difference between the VD and CS infants was

291 the higher proportion of Bacteroidetes in VD infants during the first twelve months

292 (significantly higher at one week, three months and twelve months; Supplementary Table 3).

293

294 The maternal microbiota resembled the typical adult flora as demonstrated in several previous

295 studies $[18,29,30]$ and was independent of delivery mode. The Firmicutes was the

296 dominating phylum, representing $74 \%$ and $71 \%$ in mean relative abundance for the $15 \mathrm{VD}$

297 and nine CS mothers respectively, followed by Bacteroidetes (16\% and 13\% respectively),

298 Actinobacteria (7\% and 12\% respectively), Proteobacteria (3\% and 2\% respectively) and

299 Verrucomicrobia (1\% and 2\% respectively) (Figure 1, Supplementary Table 3).

300

301 The relative abundance of the major genera found in the infants and mothers are illustrated in

302 Figure 2A-B (see also Supplementary Table 4). Infants in the VD group were colonized by

303 Bacteroides to a greater extent than in the CS group (significantly more at one week [11/15 in

12

http://mc.manuscriptcentral.com/gut 
304 VD vs. $1 / 9$ in CS; Fischer's exact test $P=0.005]$, three months $[11 / 15$ vs. $1 / 9 ; P=0.005]$ and

305 twelve months $[14 / 15$ vs. $4 / 9 ; P=0.015])$. At one month of age Bifidobacterium dominated

306 the microbiota in both groups. The Enterococcus genus was found in significantly higher

307 relative abundance in the CS compared to the VD infants at one month $(P<0.0001$;

308 Supplementary Table 4). Following six months of age there was a gradual increase in

309 previous low abundant genera in both VD and CS infants. At 24 months of age, Bacteroides

310 and several genera belonging to the Clostridia class, for example Ruminococcus, a dominant

311 member of the adult microbiota also dominated the infant microbiota.

312

313 The gradual shift in community composition was accompanied by an increase in $\alpha$-diversity

314 over time, with a significant increase in Shannon diversity index between each pair of

315 succeeding time points from three months of age and onward (Figure 3). Similar results were

316 obtained for evenness (Pielou's index; Supplementary figure 1A) and estimated richness

317 (Chao1; Supplementary figure 1B). The low increase in diversity during the first three

318 months may be related to that most (83\%) infants were exclusively breast fed up to this age

319 (Supplementary Table 1). CS delivery was associated with significantly lower total

320 microbiota diversity when considering all time points in the infants $(P=0.047$ with repeated

321 measures ANOVA; Supplementary table 5). At individual time points, the total microbiota

322 diversity was significantly lower in the CS delivered infants at twelve months

323 (Supplementary Table 5; Figure 3). The total microbiota diversity did not differ significantly

324 between the VD and CS mothers (Supplementary Table 6). Narrowing the analysis to the

325 phylum level, CS delivery was associated with a lower diversity of the Bacteroidetes phylum

326 when considering all time points $(P=0.002$; Supplementary table 5$)$. For individual time

327 points the Bacteroidetes diversity was significantly lower in the CS infants than VD infants at

328 one, three, twelve and 24 months (Figure 4; Supplementary Table 6). The other phyla, 
329 Firmicutes, Proteobacteria, and Actinobacteria did not display any consistent differences in

330 Shannon diversity between the groups, although diversity of Firmicutes was significantly

331 lower at twelve months and Proteobacteria at 24 months in the CS infants (Supplementary

332 Table 6).

333

334 A Principal Coordinates Analysis (PCoA) plot based on pair-wise sample community

335 differences calculated with UniFrac [23] is illustrated in Figure 5. As shown in a previous

336 study [31] the microbial communities became more uniform across infants over time, which

337 is also evident when comparing the distributions of pair-wise community differences at each

338 time point (Supplementary figure 2). By 24 months of age, the communities closely

339 resembled those of the mothers (Figure 5). Although community composition converged to

340 an adult-type microbiota, diversity estimates were still significantly lower at 24 months than

341 in the mothers (Figure 3; Supplementary figure 1). Birth weight, antibiotic intake during the

342 time course, sex, and breast-feeding had no apparent impact on the microbiota composition at

343 any time point (Supplementary figures 3-6).

344

345 In order to investigate possible mother-to-child transmission of bacteria, the presence of

346 specific unique sequences (sequence types) was compared between infants and their mothers,

347 as well as between infants and other mothers. For each mother-child pair, we calculated the

348 fraction of the sequence types found in the child that were also found in the mother (number

349 of shared sequence types/number of child sequence types). The VD infants shared a

350 significantly higher proportion of sequence types with their own mother than with the other

351 mothers at one week and 24 months when considering all bacterial taxa (Supplementary

352 Table 7). Considering one phylum at a time, Bacteroidetes displayed a significantly higher 
overlap at one and six months, and Firmicutes at six and 24 months. No significant overlap

was observed for the CS delivered infants at any time point.

355

Caesarean section was associated with moderately lower levels of the Th1-associated

CCL22 did not differ significantly between the birth modes.

Table 1. Repeated measures ANOVAs to test whether there were any significant differences in CXCL10 and CXCL11 levels during the first two years of life between caesarean (CS) and vaginally (VD) delivered infants. The ANOVAs where calculated on log chemokine levels.

363

364 $\mathrm{n}=$ number of infants.

\begin{tabular}{|l|l|l|l|l|l|l|l|l|l|l|l|l|l|l|l|}
\hline & & \multicolumn{9}{|c|}{ CXCL10 (mean, pg/ml) } & \multicolumn{7}{c|}{ CXCL11 (mean, pg/ml) } \\
\hline Birth mode & & $6 \mathrm{~m}$ & $\mathrm{n}$ & $12 \mathrm{~m}$ & $\mathrm{n}$ & $24 \mathrm{~m}$ & $\mathrm{n}$ & $\mathrm{p}^{*}$ & $6 \mathrm{~m}^{* *}$ & $\mathrm{n}$ & $12 \mathrm{~m}$ & $\mathrm{n}$ & $24 \mathrm{~m}$ & $\mathrm{n}$ & $\mathrm{p}^{*}$ \\
\hline VD & $\mathrm{n}=14$ & 97 & 9 & 116 & 13 & 112 & 13 & 0.05 & 529 & 9 & 500 & 13 & 527 & 13 & 0.008 \\
\hline CS & $\mathrm{n}=7$ & 37 & 4 & 166 & 3 & 71 & 4 & & 49 & 4 & 347 & 3 & 518 & 4 & \\
\hline
\end{tabular}

*= Repeated measures ANOVA including all time points (six, twelve, and 24 months). Because the non-normal distribution, the values were log transformed before. One VD infant and two CS infants where not measured at any time point and were hence excluded from the analysis. For the remaining subjects, when a sample was missing at a specific age, the value corresponding to the median value for the specific chemokine at that age group was given before repeated measures ANOVA was performed.

$* *=p<0.001$ with student $t$-test after log transformation at that specific time point.

\section{DISCUSSION}

373 Microbial colonization of the infant gut gastrointestinal tract is important for the postnatal

374 development of the immune system. In this study, caesarean section delivered infants who are

375 not entering the birth canal of the mother, either lacked or displayed a delayed colonization of

376 one of the major gut phylum, the Bacteroidetes. The colonization of this phylum was delayed

377 by up to one year for some infants. The total microbiota diversity was also lower in the CS

378 infants, probably largely as a consequence of the lack of this phylum. This was not a

379 consequence of antimicrobial treatment, as none of the CS mothers were given antibiotics

380 before surgery and the microbial diversity did not differ between mothers who were given

381 antibiotics prophylactically and those who did not receive antibiotics. Comparisons of 
382 intestinal microbiota have not conclusively confirmed bacterial transmission [31, 32],

383 although the genus Bacteroides has been proposed to be transmitted from the maternal gut

$384[33,34]$. Our study corroborates earlier studies reporting a delayed colonization of

385 Bacteroides in babies delivered by CS $[4,5]$. In addition our study provides evidence that

386 specific lineages of the intestinal microbiota, as defined by 16S rRNA gene sequences, are

387 transmitted from mother to child during vaginal delivery.

388

389 It is important to note that bacterial composition changes as a consequence of freezing the

390 fecal samples $[35,36]$ and that PCR amplification can induce taxon-specific biases. However,

391 there were no significant differences in storage times between the VD and CS samples and

392 since the same primer pair and PCR conditions were used for all samples, these effects should

393 not contribute to the observed differences in microbiota composition and alpha-diversity

394 between the sample groups.

395

396 The genus Enterococcus, which is a typical fecal bacterium, is usually acquired during the

397 first week of life [37]. Colonization has previously not been shown to depend on delivery

398 mode, suggesting other sources in addition to the maternal intestinal microbiota [5], such as

399 the environment [38] and breast milk [39]. We found that CS infants had a higher relative

400 abundance of Enterococcus at one month of age, suggesting that the lack of bacteria

401 transmitted through vaginal delivery favors the growth and colonization of enterococci.

402

403 Appropriate microbial stimulation during infancy is required for the development of a more

404 balanced immune phenotype, including maturation of Th1-like responses and appropriate

405 development of regulatory $\mathrm{T}$ cell responses [6, 40, 41]. It is well known that early life events

406 occurring during critical windows of immune development can have long-term impact on 
407 immune-mediated diseases such as allergy [15, 42, 43], diabetes, and inflammatory bowel

408 disease. We hypothesized that early differences in the gut microbiota could shape later

409 immune responsiveness, influencing the Th1 maturation trajectory. Our findings of a lower

410 microbial diversity in the CS infants, and lower circulating levels of the Th1-related

411 chemokines CXCL10 and CXCL11, support this view. Previous studies have shown that

412 Bacteroides fragilis exert strong effects on the immune system. This is mediated by the

413 capsular polysaccharide (PSA), which enhances T-cell mediated immune responses and

414 affects the Th1/Th2 balance [44, 45]. Furthermore, B. thetaiotamicron is also known to affect

415 the immune system [46]. Thus, the lower abundance of Bacteroides among the CS infants

416 may be a contributing factor to the observed differences in Th1-associated chemokines.

417 Future studies with larger sample sizes will be able to address the effects of individual

418 microbes on chemokine levels.

419

420 With few exceptions [31], previous studies have reported Bifidobacterium to be one of the

421 dominant genera of the early infant intestinal microbiota [4, 33, 47, 48, 49], and changes in

422 relative abundance of this genus have been related to delivery mode $[4,16,34]$. Also in the

423 present study, Bifidobacterium was the dominant genus from one to twelve months of age

424 with a gradual decline following weaning. The abundance was not affected by delivery mode,

425 however, and we could not detect any significant overlap in the mothers' and babies' rRNA

426 sequences. Hence, Bifidobacterium could primarily be transmitted from the breast milk, and

427 to a lesser extent from the intestinal micobiota, as suggested but not confirmed previously $428 \quad[50]$.

429

430 In accordance with recent studies [31, 47, 48], our results demonstrate considerable

431 individual differences in the microbial succession during the first year of life. This is 
432 probably a result of differences in time of weaning, and incidental exposure of bacteria from

433 the environment. Community composition converges to an adult-like state within two years.

434 However, even at two years the microbiota appears not to be fully developed, since the

435 diversity was significantly lower than in mothers. This was also evident in a recent study

436 reporting a lower microbial diversity in a 2.5 years old child than in its mother [51].

438 An association between CS delivery and the development of allergic disease has been

439 observed in several studies $[52,53]$ and a lower microbial diversity has been observed in

440 allergic infants before onset of disease [13]. We conclude that CS is associated with a lower

441 bacterial diversity during the first two years of life, a lower abundance and diversity of the

442 phylum Bacteroidetes, and lower circulating levels of Th1-associated chemokines during

443 infancy.

\section{ACKNOWLEDGEMENTS}

446 We thank Mrs Lena Lindell, Linköping, Mrs Elisabeth Andersson, Norrköping, Mrs Linnea

447 Andersson, Jönköping, and Mrs Eivor Folkesson, Motala, Dr Göran Oldaeus, Jönköping, and

448 Dr Ted Jacobsson, Linköping, for their brilliant and enthusiastic work guiding the families

449 through the study and all the sampling procedures. We also thank Ms Martina Abelius, Mrs

450 Anne-Marie Fornander and Ms Anna Forsberg in Linköping for excellent technical assistance 451

\section{COMPETING INTERESTS}

453 None. 
455 FUNDING

456 This work was supported by the Ekhaga Foundation and the Söderbergs Foundation to LE

457 and by the Swedish Research Council, the Research Council for the South-East Sweden, the

458 Swedish Asthma and Allergy Association, the Olle Engkvist Foundation, the Vårdal

459 Foundation - for Health Care Sciences and Allergy Research to MJ and by the Swedish

460 Research Councils VR and FORMAS to AA. KH was funded through a direct grant from

461 Unilever.

462

463

464

465

466

467

468

469

470

471

472

473

474

475

476

477

478

19

http://mc.manuscriptcentral.com/gut 


\section{REFERENCES}

4801 Ley RE, Peterson DA, Gordon JI. Ecological and evolutionary forces shaping

481 microbial diversity in the human intestine. Cell 2006;124:837-48.

4822 Dominguez-Bello MG, Costello EK, Contreras M, et al. Delivery mode shapes

483 the acquisition and structure of the initial microbiota across multiple body habitats in

484 newborns. Proc Natl Acad Sci U S A 2010;107:11971-5.

4853 Sufang G, Padmadas SS, Fengmin Z, et al. Delivery settings and caesarean

486 section rates in China. Bull World Health Organ 2007;85:755-62.

4874 Penders J, Thijs C, Vink C, et al. Factors influencing the composition of the

488 intestinal microbiota in early infancy. Pediatrics 2006;118:511-21.

$4895 \quad$ Adlerberth I, Lindberg E, Åberg N, et al. Reduced enterobacterial and increased

490 staphylococcal colonization of the infantile bowel: an effect of hygienic lifestyle? Pediatr Res

$491 \quad 2006 ; 59: 96-101$.

4926 McLoughlin RM, Mills KH. Influence of gastrointestinal commensal bacteria

493 on the immune responses that mediate allergy and asthma. J Allergy Clin Immunol 2011.

4947 Eggesbö M, Botten G, Stigum H, et al. Is delivery by cesarean section a risk

495 factor for food allergy? J Allergy Clin Immunol 2003;112:420-6.

4968 Bager P, Wohlfahrt J, Westergaard T. Caesarean delivery and risk of atopy and

497 allergic disease: meta-analyses. Clin Exp Allergy 2008;38:634-42.

4989 Watanabe S, Narisawa Y, Arase S, et al. Differences in fecal microflora

499 between patients with atopic dermatitis and healthy control subjects. J Allergy Clin Immunol

$500 \quad 2003 ; 111: 587-91$.

$501 \quad 10 \quad$ Björkstén B, Naaber P, Sepp E, et al. The intestinal microflora in allergic

502 Estonian and Swedish 2-year-old children. Clinical and Experimental Allergy 1999;29:1-5.

$50311 \quad$ Wang M, Karlsson C, Olsson C, et al. Reduced diversity in the early fecal

504 microbiota of infants with atopic eczema. J Allergy Clin Immunol 2008;121:129-34.

$50512 \quad$ Forno E, Onderdonk AB, McCracken J, et al. Diversity of the gut microbiota

506 and eczema in early life. Clin Mol Allergy 2008;6:11.

50713 Abrahamsson TR, Jakobsson HE, Andersson AF, et al. Low diversity of the gut

508 microbiota in infants with atopic eczema. J Allergy Clin Immunol 2012;129:434-40, 40 e1-2.

$50914 \quad$ Böttcher MF, Jenmalm MC, Björkstén B. Immune responses to birch in young

510 children during their first 7 years of life. Clin Exp Allergy 2002;32:1690-8.

$511 \quad 15 \quad$ Jenmalm MC. Childhood immune maturation and allergy development:

512 regulation by maternal immunity and microbial exposure. Am J Reprod Immunol 2011;66

513 Suppl 1:75-80.

$51416 \quad$ Huurre A, Kalliomäki M, Rautava S, et al. Mode of delivery - effects on gut

515 microbiota and humoral immunity. Neonatology 2008;93:236-40.

$51617 \quad$ Abrahamsson TR, Jakobsson T, Böttcher MF, et al. Probiotics in prevention of

517 IgE-associated eczema: a double-blind, randomized, placebo-controlled trial. J Allergy Clin

518 Immunol 2007;119:1174-80.

51918 Andersson AF, Lindberg M, Jakobsson H, et al. Comparative analysis of human

520 gut microbiota by barcoded pyrosequencing. PLoS ONE 2008;3:e2836.

$521 \quad 19 \quad$ Herlemann DP, Labrenz M, Jurgens K, et al. Transitions in bacterial

522 communities along the $2000 \mathrm{~km}$ salinity gradient of the Baltic Sea. Isme J 2011.

$52320 \quad$ Klindworth A, Pruesse E, Schweer T, et al. Evaluation of general 16S

524 ribosomal RNA gene PCR primers for classical and next-generation sequencing-based

525 diversity studies. Nucleic Acids Res 2013;41:e1.

$52621 \quad$ Quince C, Lanzen A, Davenport RJ, et al. Removing noise from pyrosequenced

527 amplicons. BMC Bioinformatics 2011;12:38. 
52822 Maidak BL, Cole JR, Lilburn TG, et al. The RDP (Ribosomal Database Project) 529 continues. Nucleic Acids Res 2000;28:173-4.

53023 Hamady M, Knight R. Microbial community profiling for human microbiome

531 projects: Tools, techniques, and challenges. Genome Res 2009;19:1141-52.

$532 \quad 24 \quad$ Hayek LCaMAB. Surveying natural populations. New York: Columbia

533 University Press 1996.

$53425 \quad$ Pielou EC. The measurement of diversity in different types of biological

535 collections. J Theor Biol 1966;13:131-44.

$53626 \quad$ Chao A. Non-parametric estimation of the number of classes in a population.

537 Scand J Stat 1984;11:265-70.

53827 de Jager W, Prakken BJ, Bijlsma JW, et al. Improved multiplex immunoassay

539 performance in human plasma and synovial fluid following removal of interfering

540 heterophilic antibodies. J Immunol Methods 2005;300:124-35.

$54128 \quad$ Abrahamsson TR, Abelius M, Forsberg A, et al. A Th1/Th2-associated

542 chemokine imbalance during infancy in children developing eczema, wheeze and

543 sensitization. Clin Exp Allergy 2011.

54429 Dethlefsen L, Huse S, Sogin ML, et al. The pervasive effects of an antibiotic on

545 the human gut microbiota, as revealed by deep 16S rRNA sequencing. PLoS Biol

$546 \quad 2008 ; 6: \mathrm{e} 280$.

$54730 \quad$ Eckburg PB, Bik EM, Bernstein CN, et al. Diversity of the human intestinal

548 microbial flora. Science 2005;308:1635-8.

$54931 \quad$ Palmer C, Bik EM, Digiulio DB, et al. Development of the Human Infant

550 Intestinal Microbiota. PLoS Biol 2007;5:e177.

55132 Turnbaugh PJ, Hamady M, Yatsunenko T, et al. A core gut microbiome in

552 obese and lean twins. Nature 2009;457:480-4.

$553 \quad 33 \quad$ Vaishampayan PA, Kuehl JV, Froula JL, et al. Comparative metagenomics and

554 population dynamics of the gut microbiota in mother and infant. Genome Biol Evol

555 2010;2010:53-66.

$55634 \quad$ Adlerberth I, Strachan DP, Matricardi PM, et al. Gut microbiota and

557 development of atopic eczema in 3 European birth cohorts. J Allergy Clin Immunol

$558 \quad 2007 ; 120: 343-50$.

$55935 \quad$ Maukonen J, Simoes C, Saarela M. The currently used commercial DNA-

560 extraction methods give different results of clostridial and actinobacterial populations derived

561 from human fecal samples. FEMS Microbiol Ecol 2012;79:697-708.

$56236 \quad$ Bahl MI, Bergstrom A, Licht TR. Freezing fecal samples prior to DNA

563 extraction affects the Firmicutes to Bacteroidetes ratio determined by downstream

564 quantitative PCR analysis. FEMS Microbiol Lett 2012;329:193-7.

$56537 \quad$ Rotimi VO, Duerden BI. The development of the bacterial flora in normal

566 neonates. J Med Microbiol 1981;14:51-62.

$56738 \quad$ Singh N, Leger MM, Campbell J, et al. Control of vancomycin-resistant

568 enterococci in the neonatal intensive care unit. Infect Control Hosp Epidemiol 2005;26:646-

5699.

57039 Martin R, Langa S, Reviriego C, et al. Human milk is a source of lactic acid

571 bacteria for the infant gut. J Pediatr 2003;143:754-8.

$57240 \quad$ Lloyd CM, Hawrylowicz CM. Regulatory T cells in asthma. Immunity

$573 \quad 2009 ; 31: 438-49$.

$57441 \quad$ Vuillermin PJ, Ponsonby AL, Saffery R, et al. Microbial exposure, interferon

575 gamma gene demethylation in naive T-cells, and the risk of allergic disease. Allergy

$576 \quad 2009 ; 64: 348-53$. 
$57742 \quad$ Björkstén B. The intrauterine and postnatal environments. J Allergy Clin 578 Immunol 1999;104:1119-27.

$57943 \quad$ Prescott SL. Early origins of allergic disease: a review of processes and

580 influences during early immune development. Curr Opin Allergy Clin Immunol 2003;3:125-

$581 \quad 32$.

58244 Mazmanian SK, Liu CH, Tzianabos AO, et al. An immunomodulatory molecule 583 of symbiotic bacteria directs maturation of the host immune system. Cell 2005;122:107-18.

58445 Mazmanian SK, Round JL, Kasper DL. A microbial symbiosis factor prevents

585 intestinal inflammatory disease. Nature 2008;453:620-5.

$58646 \quad$ Kelly D, Campbell JI, King TP, et al. Commensal anaerobic gut bacteria

587 attenuate inflammation by regulating nuclear-cytoplasmic shuttling of PPAR-gamma and

588 RelA. Nat Immunol 2004;5:104-12.

$58947 \quad$ Eggesbö M, Moen B, Peddada S, et al. Development of gut microbiota in

590 infants not exposed to medical interventions. APMIS 2011;119:17-35.

$59148 \quad$ Tanya Yatsunenko FER, Mark J. Manary, Indi Trehan, Maria Gloria

592 Dominguez-Bello5, Monica Contreras, Magda Magris, Glida Hidalgo, Robert N. Baldassano,

593 Andrey P. Anokhin, Andrew C. Heath, Barbara Warner, Jens Reeder, Justin Kuczynski, J.

594 Gregory Caporaso, Catherine A. Lozupone, Christian Lauber, Jose Carlos Clemente, Dan

595 Knights, Rob Knight \& Jeffrey I. Gordon. Human gut microbiome viewed across age and

596 geography. Nature 2012.

$59749 \quad$ Turroni F, Peano C, Pass DA, et al. Diversity of Bifidobacteria within the Infant

598 Gut Microbiota. PLoS ONE 2012;7:e36957.

$59950 \quad$ Tannock GW, Fuller R, Smith SL, et al. Plasmid profiling of members of the

600 family Enterobacteriaceae, lactobacilli, and bifidobacteria to study the transmission of

601 bacteria from mother to infant. J Clin Microbiol 1990;28:1225-8.

$60251 \quad$ Koenig JE, Spor A, Scalfone N, et al. Succession of microbial consortia in the

603 developing infant gut microbiome. Proc Natl Acad Sci U S A 2011;108 Suppl 1:4578-85.

$60452 \quad$ Salam MT, Margolis HG, McConnell R, et al. Mode of delivery is associated

605 with asthma and allergy occurrences in children. Ann Epidemiol 2006;16:341-6.

$60653 \quad$ Thavagnanam S, Fleming J, Bromley A, et al. A meta-analysis of the

607 association between Caesarean section and childhood asthma. Clin Exp Allergy 2008;38:629-

60833.

609

610

611

612

613

614

615

616

617

618 
620 Figure 1. Phylum level microbiota composition in mothers and their infants and at one week, 621 one, three, six, twelve, and 24 months. The mean relative abundances (\%) of the most 622 abundant bacterial phyla in the 15 VD infants (A) and nine CS infants (B), as well as in their 623 mothers are shown.

624

625 Figure 2. Genus level microbiota composition in mothers and their infants and at one week, 626 one, three, six, twelve, and 24 months. The mean relative abundances (\%) of the most 627 abundant bacterial genera in the $15 \mathrm{VD}$ infants (A) and nine CS infants (B), as well as in their 628 mothers are shown. Only genera comprising $\geq 1 \%$ of the total community were included.

629 Abbreviations: Pr, Proteobacteria; Fi, Firmicutes; Ba, Bacteroides; Ac, Actinobacteria. 630

631 Figure 3. Increase in fecal microbiota alpha-diversity over time. Distributions of Shannon 632 diversity indices displayed for the 15 VD infants and 9 CS infants at one week, one, three, 633 six, twelve, and 24 months, and for their mothers. Fifty percent of the data points reside 634 within boxes, $75 \%$ within whiskers, and median values are indicated by horizontal lines 635 within boxes (circles indicate individual values). Wilcoxon signed rank tests were conducted 636 to compare Shannon diversity between adjacent time points, and Wilcoxon rank-sum tests to 637 compare diversity between delivery modes within time points; *** indicates $P<0.001$, ** 638 indicates $P<0.01$ and $*$ indicates $P<0.05$.

639

640 Figure 4. Increase in Bacteroidetes alpha-diversity over time. Distributions of Shannon 641 diversity indices displayed for the 15 VD infants and 9 CS infants at one week, one, three, 642 six, twelve, and 24 months, and for their mothers. *** indicates $P<0.001$, ** indicates $P<$ 6430.01 and * indicates $P<0.05$. Fifty percent of the data points reside within boxes, $75 \%$ 644 within whiskers, and median values are indicated by horizontal lines within boxes (circles 645 indicate individual values).

647 Figure 5. Individuality and convergence of infant microbiota. Principal Co-ordinates 648 Analysis (PCoA) was performed on all pair-wise community differences (calculated with 649 UniFrac (Hamady M, 2009)), and samples from infants at one week, one, three, six, twelve, 650 and 24 months, and from the mothers are highlighted in the different boxes. VD and CS 651 infants/mothers are displayed in red and blue, respectively. 
Figure 1.

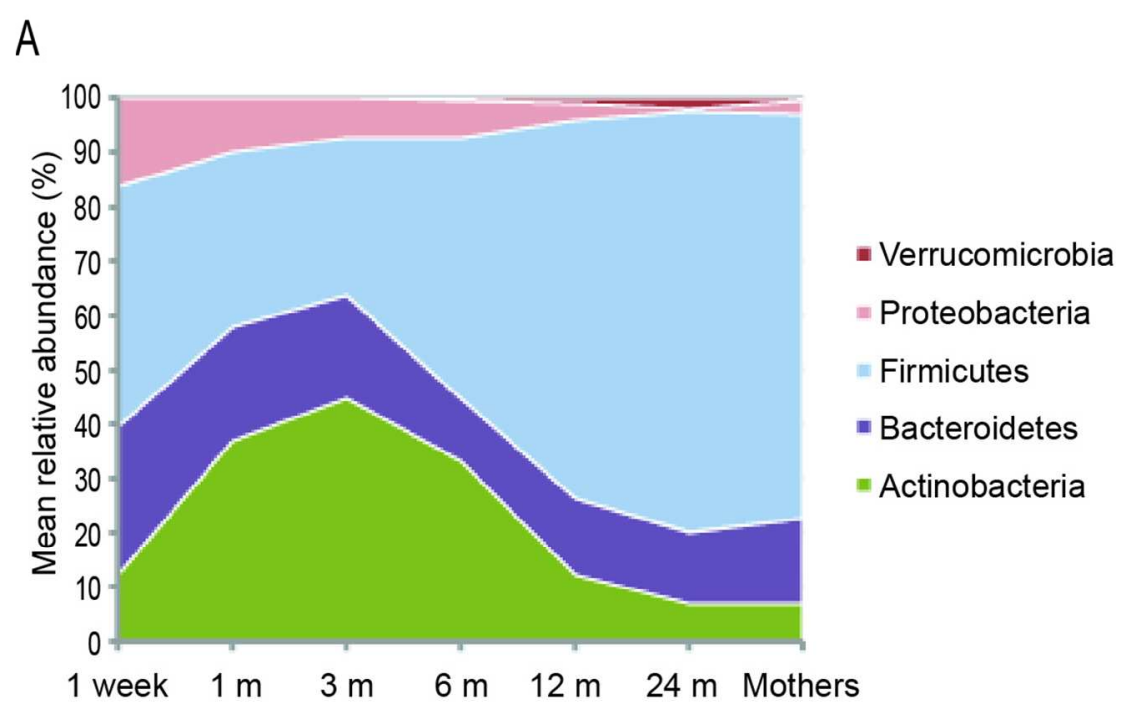

B

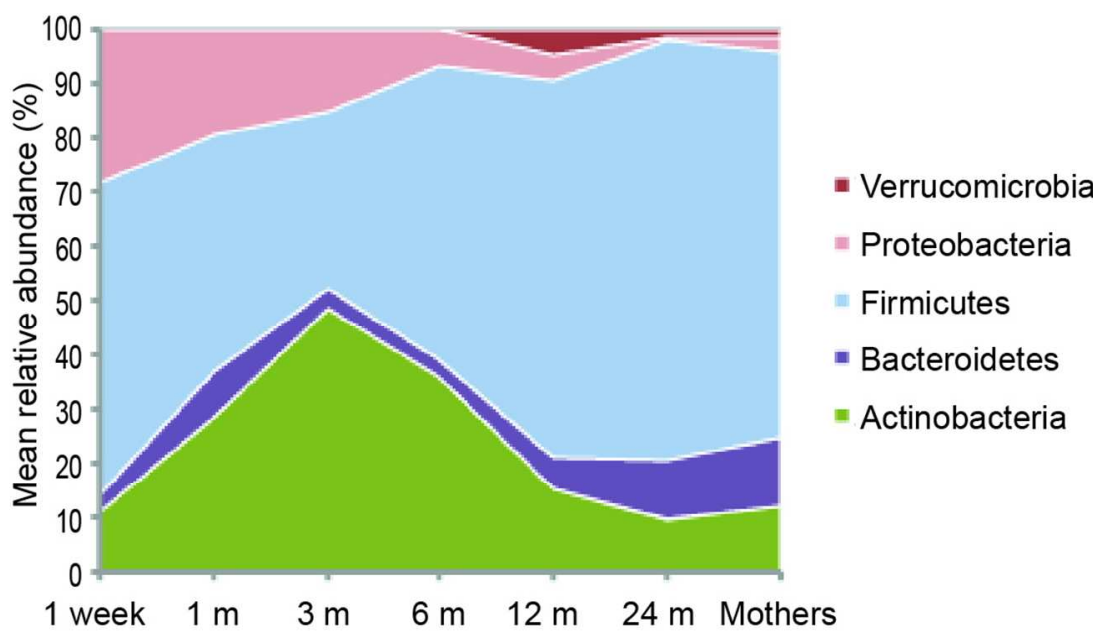

$396 \times 562 \mathrm{~mm}(72 \times 72 \mathrm{DPI})$

http://mc.manuscriptcentral.com/gut 
Figure 2.

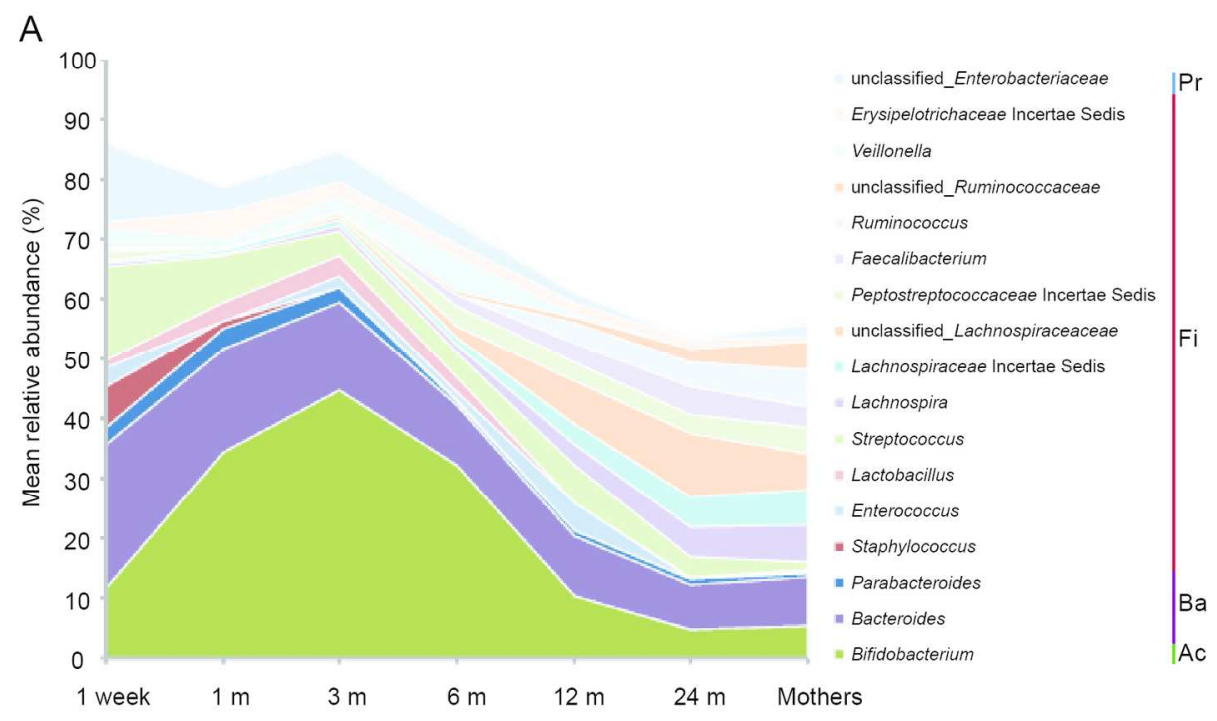

B

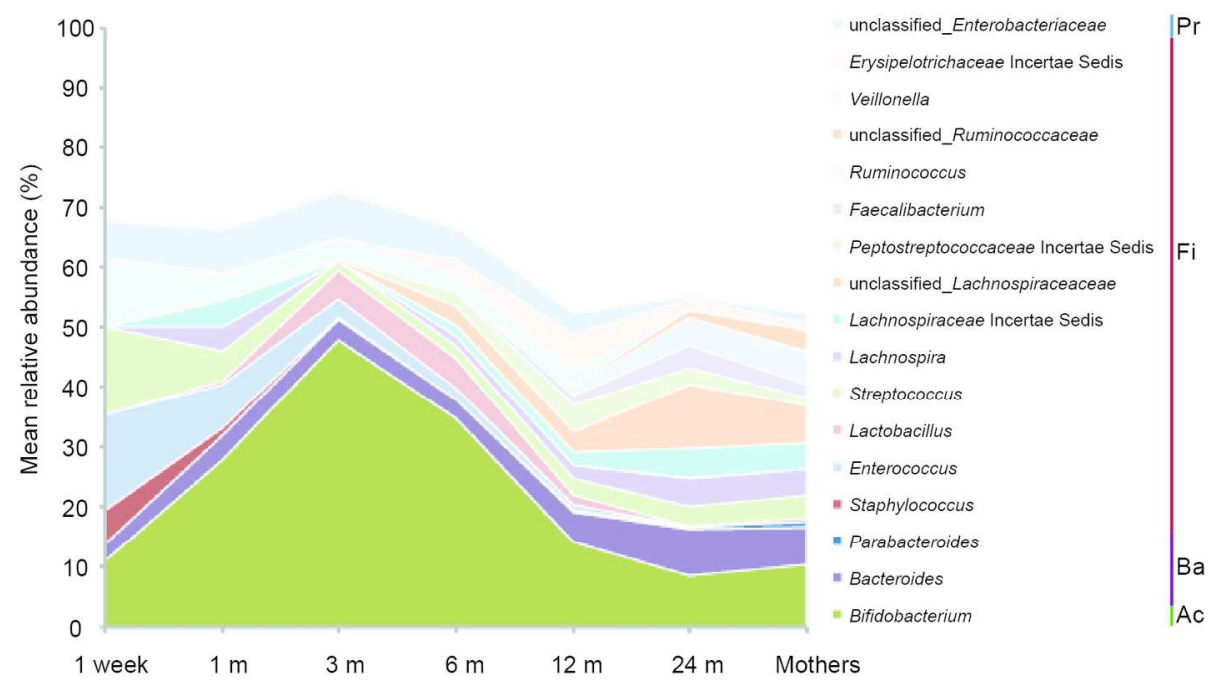

$141 \times 185 \mathrm{~mm}(300 \times 300$ DPI $)$ 
Figure 3.

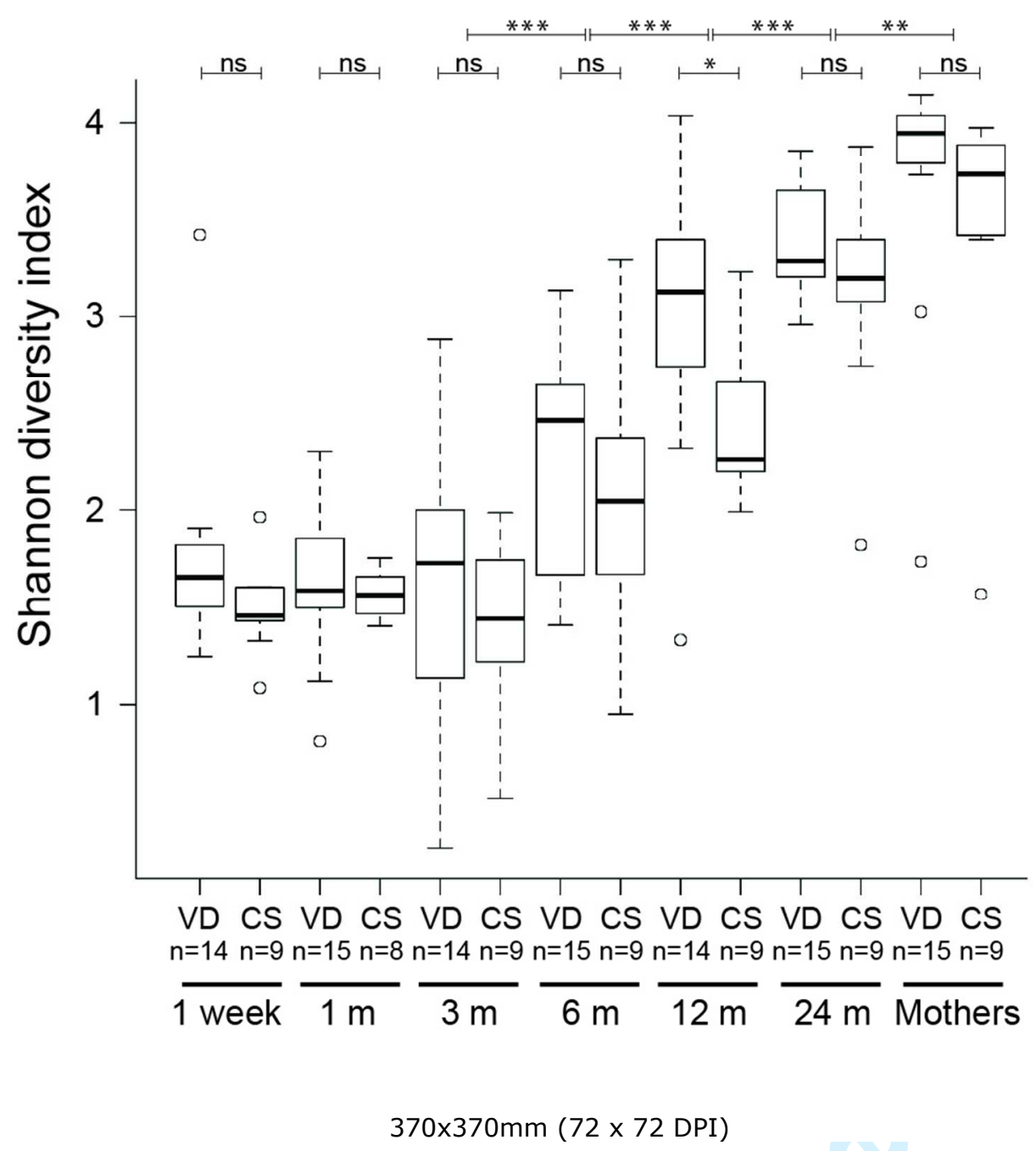

http://mc.manuscriptcentral.com/gut 
Figure 4.

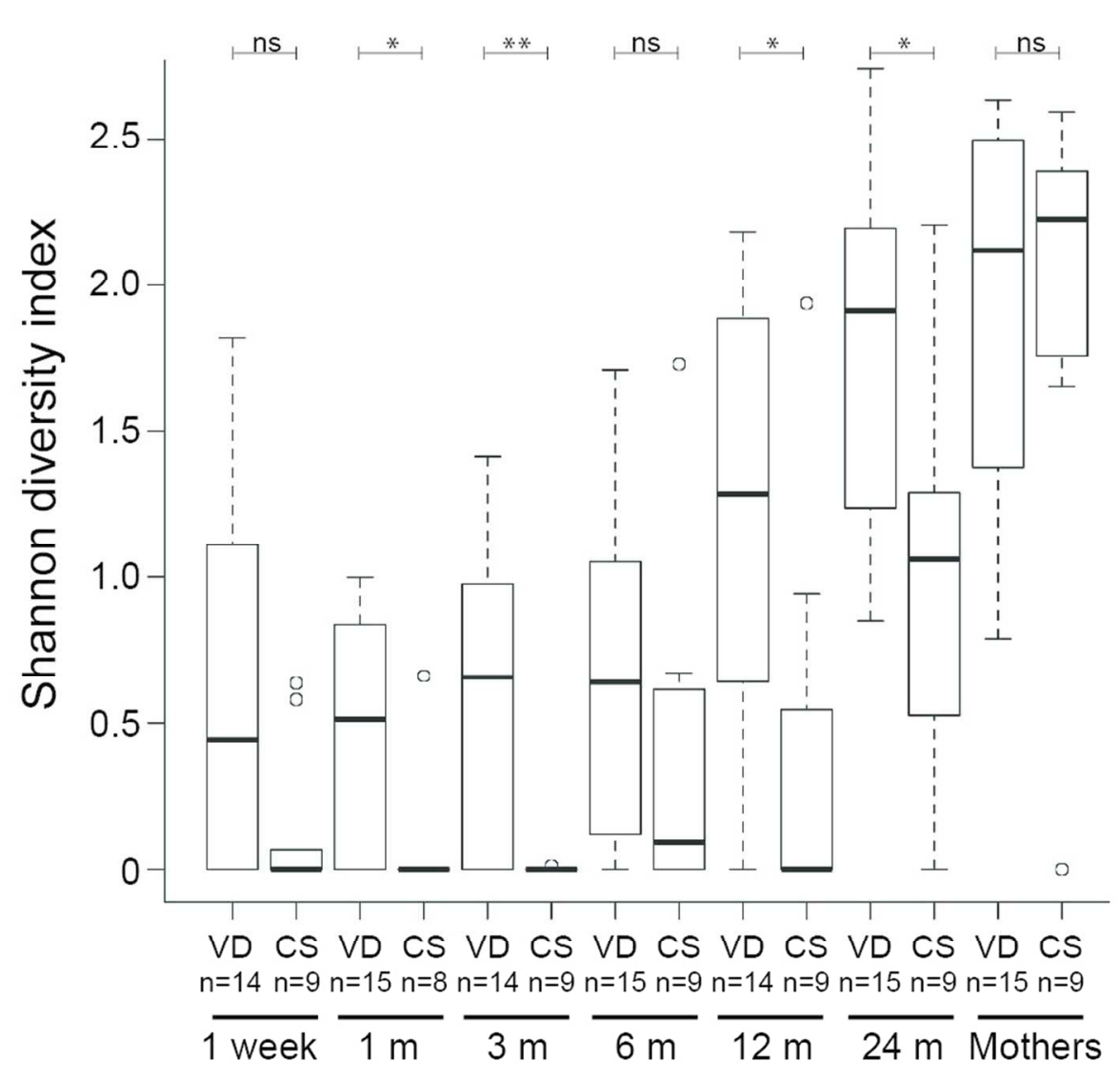

$94 \times 90 \mathrm{~mm}(300 \times 300$ DPI $)$ 


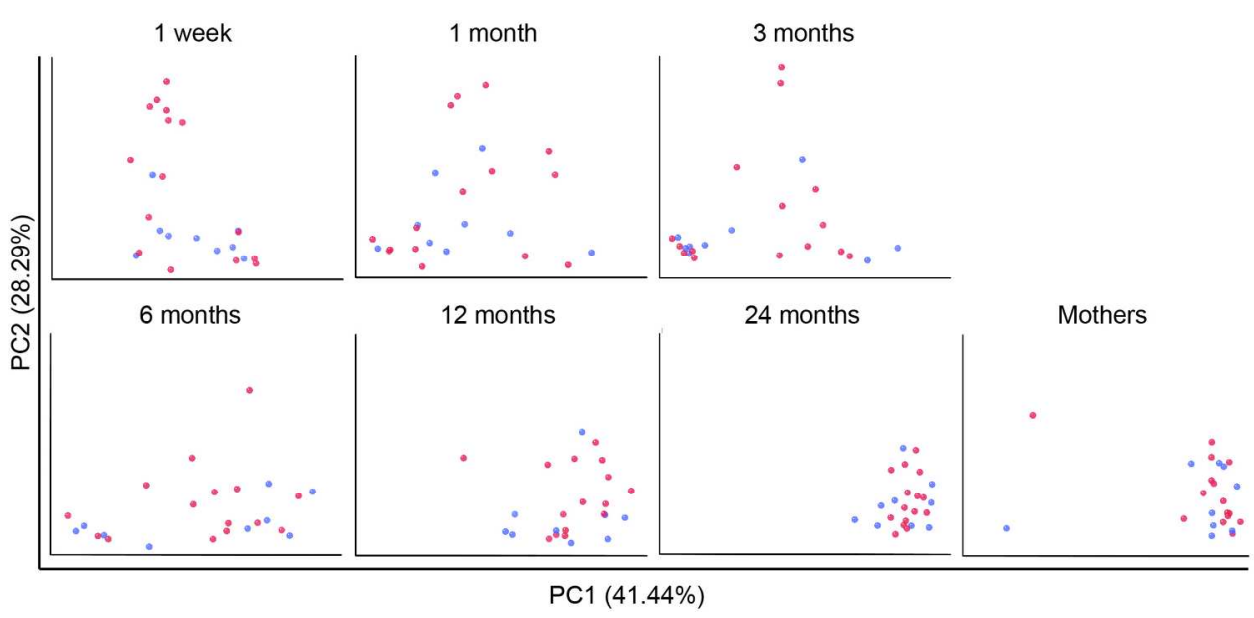

$772 \times 350 \mathrm{~mm}(72 \times 72$ DPI $)$

1

2

3

4

5

7

8

9

10

11

12

13

15

17

18

19

20

21

22

23

24

25

26

27

28

29

30

31

32

33

34

35

36

37

38

39

40

41

42

43

44

45

46

47

48

49

50

51

52

53

54

55

56

57

58

59

60

http://mc.manuscriptcentral.com/gut 
B
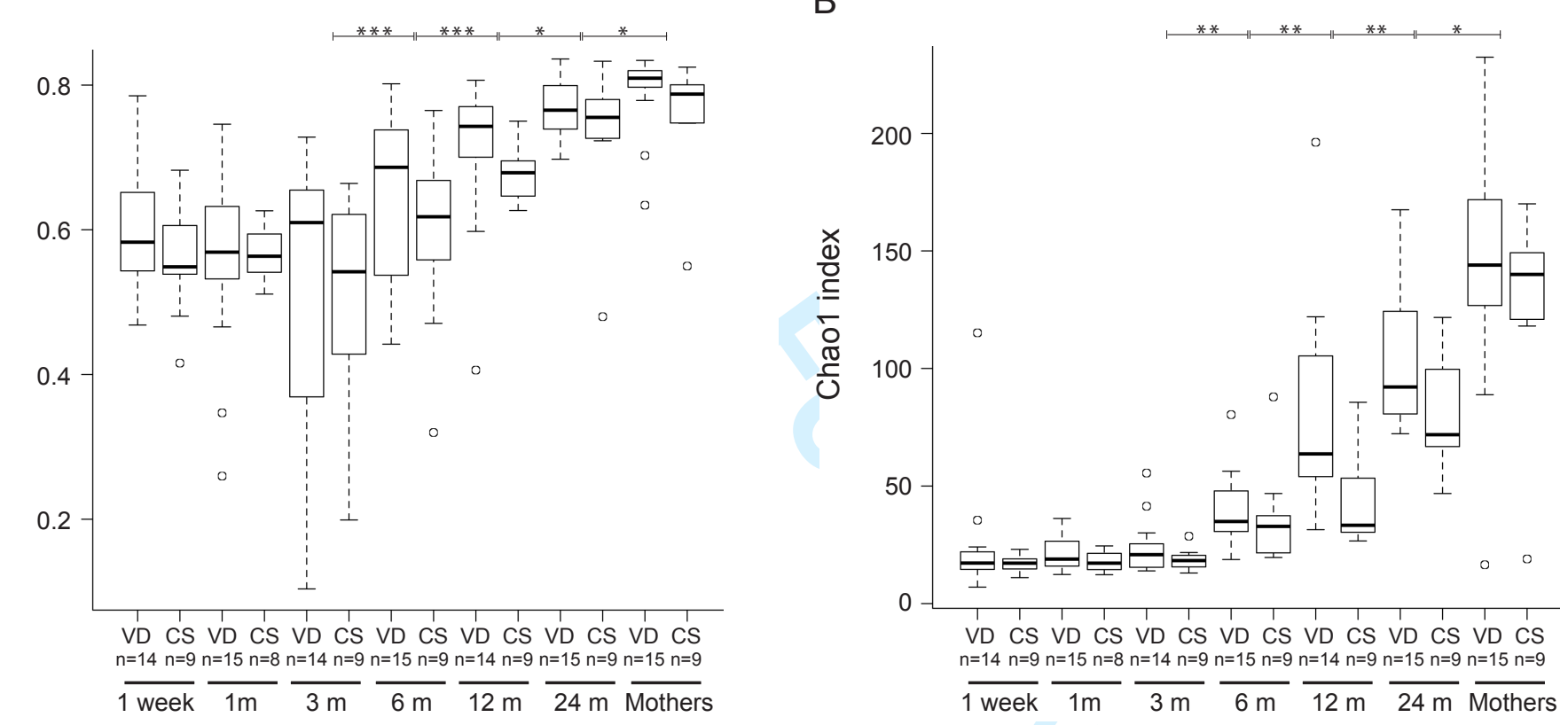

Supplementary figure 1. Pielou's evenness index $(A)$ and Chao1 index (B) values for all 24 infants as well as for the 24 mothers. Statististical significance was measured using Wilcoxon signed rank test and * indicates $P<0.05,{ }^{* *}$ indicates $\mathrm{P}<0.01,{ }^{* * *}$ indicates $\mathrm{P}<0.001$. 


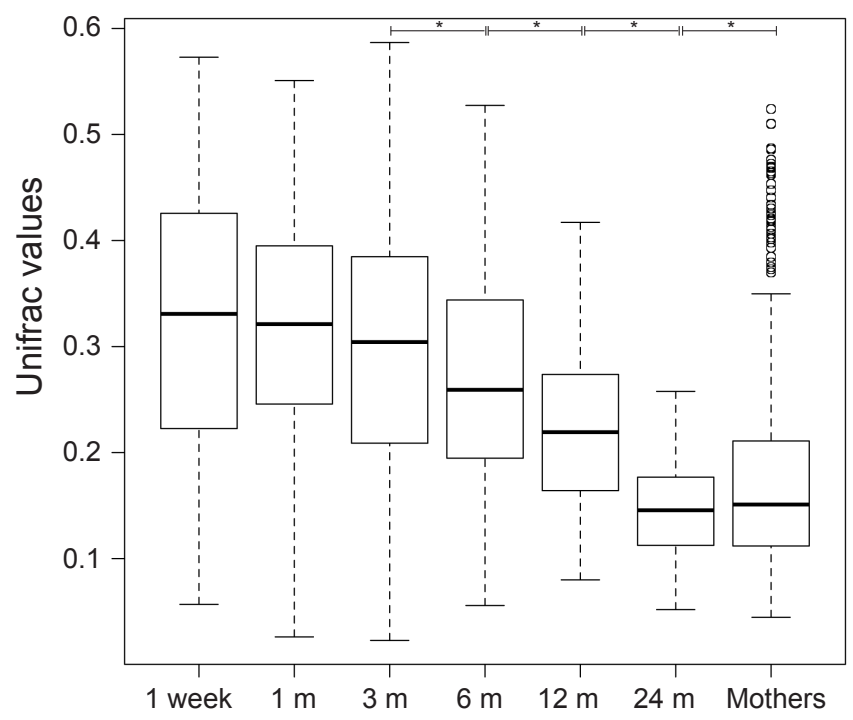

Supplementary figure 2. Pair-wise community differences. Distributions of Unifrac distance values for all 24 infants as well as for the 24 mothers. Overall, inter-subject differences declined significantly over time (Spearman rank order correlation $r=-0.45$, $P<2.2 e-16)$. There was also significant differences between adjacent time points from three months and onward; stars indicate Wilcoxon signed rank test $P<10-4$. 


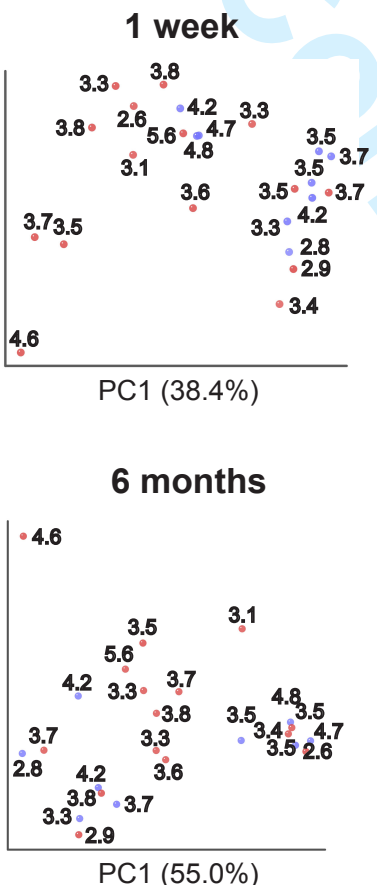

3 months
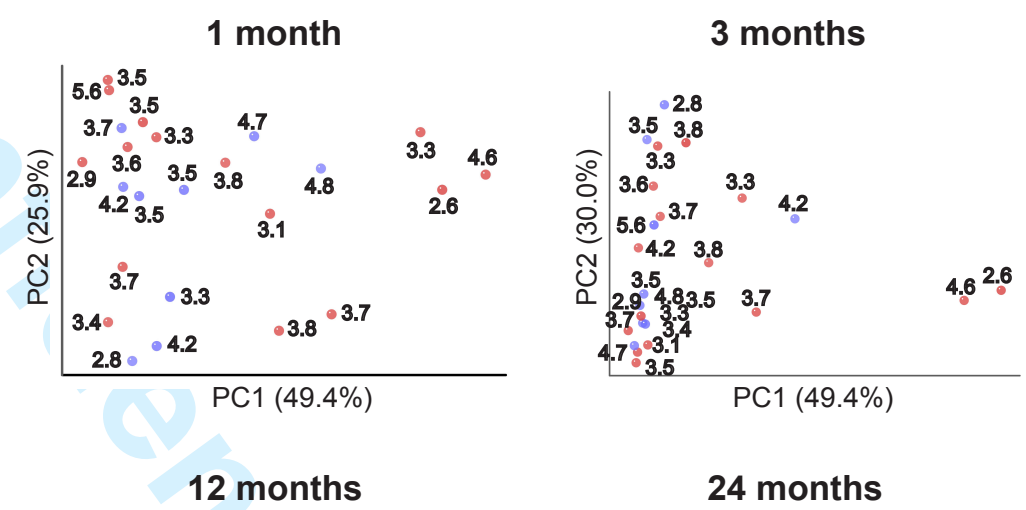

24 months

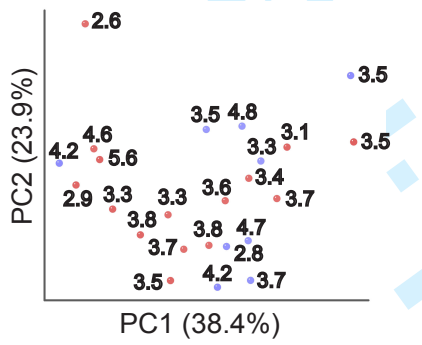

Supplementary figure 3. Principal Coordinates Analysis (PCoA) was performed on pair-wise community differences at the different time points (calculated with UniFrac (Hamady M, 2009)), and samples from all 24 infants are highlighted in the different boxes. Vaginal delivered infants are highlighted in red and CS infants are displayed in blue. Birth weights (kg) are indicated in the figure. 
Gut

3 months
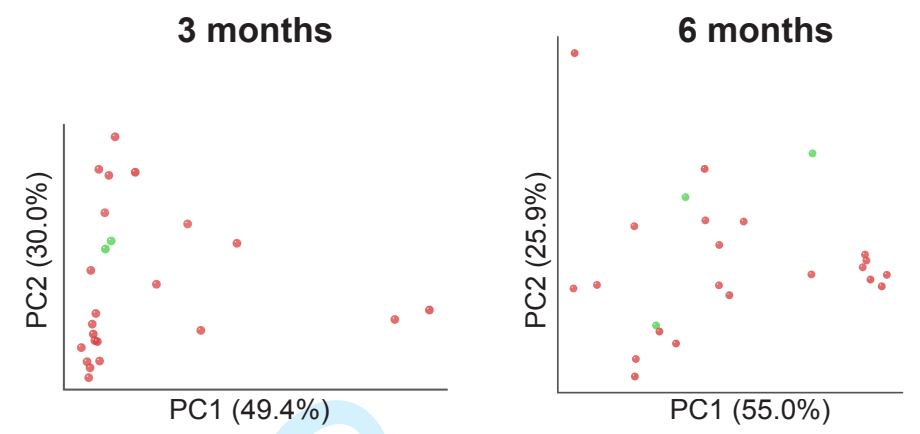

12 months

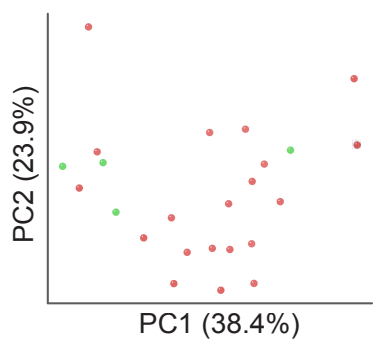

\section{4 months}

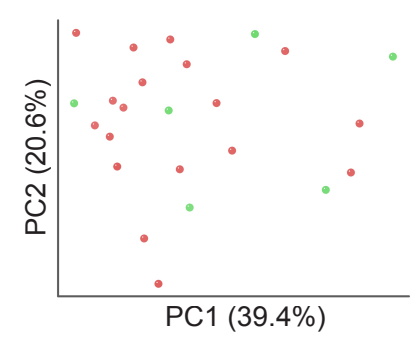

10

11 Supplementary figure 4. Principal Coordinates Analysis (PCoA) was performed on pair-wise community

12 differences at the different time points (calculated with UniFrac (Hamady M, 2009)), and samples from all infants 13 are highlighted in the different boxes. All VD and CS are displayed in red except those infants that received antibiotics 14 that are displayed in green.

15

16

17

18

19

20

21

22

23

24

25

26 


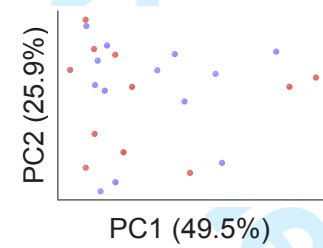

PC1 (28.14\%)

6 months

12 months
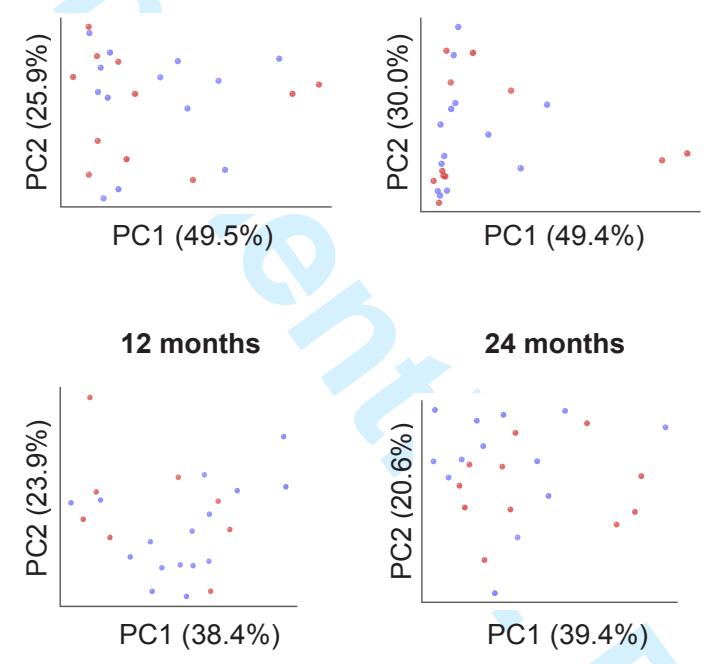

31 upplementary figure 5. Principal Coordinates Analysis (PCoA) was 32 3 (calculated with UniFrac (Hamady M, 2009)), and samples from all 24 infants 34 re highlighted in the different boxes. Males are displayed in blue and 36 males are displayed in red. 
1 week PC1 (28.14\%)
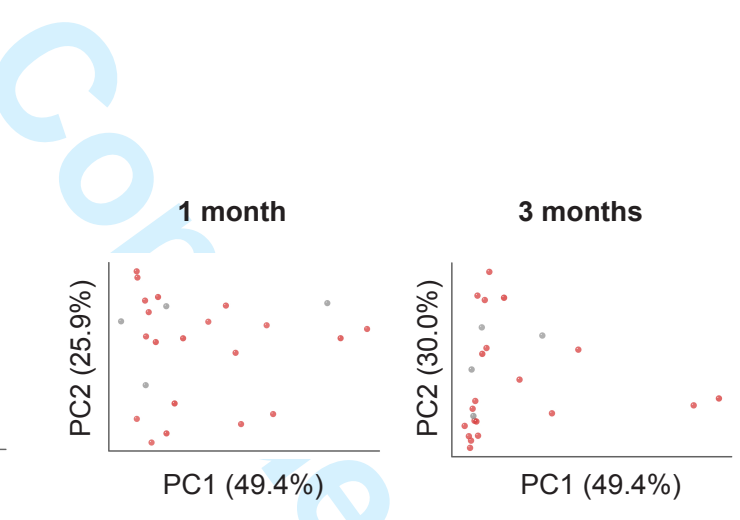

21

PC1 $(49.4 \%)$

¿2upplementary figure 6. Principal Coordinates Analysis (PCoA) was 2 2̧ 24d three months (calculated with UniFrac (Hamady M, 2009)), and samples $250 \mathrm{~m}$ all 24 infants are highlighted in the different boxes. All VD and CS that z(Bere exlusively breast-fed are displayed in red except those infants that were एकartially breast-fed from birth that are displayed in grey.

28
29
30
31
32
33
34
35
36
37
38
39
40
41
42
43
44
45
46
47
48
49
50
51
52
53
54
55
56
57
58
59
60


Supplementary Table 1. Descriptive data of the infants included in the study. Abbreviations: VD, vaginal delivery; CS, caesarean delivery; PcV, Penicillis V; Amp, Ampicillin derivative; Her, Heracillin.

\begin{tabular}{|c|c|c|c|c|c|}
\hline Baby & Sex & Delivery & $\begin{array}{l}\text { Birth } \\
\text { weights } \\
\text { (grams) }\end{array}$ & Antimicrobials & $\begin{array}{l}\text { Length of exclusive } \\
\text { and partial breast- } \\
\text { feeding (months) }\end{array}$ \\
\hline Infant $1 \mathrm{VD}$ & Male & Vaginal & 3740 & & 3,8 \\
\hline Infant 2 VD & Female & Vaginal & 2590 & & 3,12 \\
\hline Infant 3 VD & Female & Vaginal & 2970 & & 0,5 \\
\hline Infant 4 VD & Female & Vaginal & 4620 & & 3,12 \\
\hline Infant 5 VD & Female & Vaginal & 3710 & & 0,5 \\
\hline Infant 6 VD & Male & Vaginal & 3800 & & 3,8 \\
\hline Infant $7 \mathrm{VD}$ & Male & Vaginal & 5590 & $\begin{array}{l}3 \text { mo PcV, Amp, } 10 \text { mo Amp, } 13 \text { mo } \\
\text { Amp, } 15 \text { mo Her, Amp, } 19 \text { mo Amp, } 24 \\
\text { mo Amp. }\end{array}$ & 3,12 \\
\hline Infant $8 \mathrm{VD}$ & Male & Vaginal & 3110 & $5 \mathrm{mo} \mathrm{PcV}, 12 \mathrm{mo} \mathrm{PcV}$ & 3,12 \\
\hline Infant 9 VD & Female & Vaginal & 3810 & & 3,8 \\
\hline Infant $10 \mathrm{VD}$ & Female & Vaginal & 3460 & $20 \mathrm{mo} \mathrm{PcV}$. & 3,12 \\
\hline Infant $11 \mathrm{VD}$ & Male & Vaginal & 3550 & & 3,10 \\
\hline Infant 12 VD & Female & Vaginal & 3400 & & 3,12 \\
\hline Infant 13 VD & Male & Vaginal & 3250 & & 0,3 \\
\hline Infant $14 \mathrm{VD}$ & Male & Vaginal & 3450 & & 3,8 \\
\hline Infant 15 VD & Female & Vaginal & 3260 & $8 \mathrm{mo} \mathrm{PcV}, 20 \mathrm{mo} \mathrm{PcV}, 21 \mathrm{mo} \mathrm{PcV}$ & 0,8 \\
\hline Infant $1 \mathrm{CS}$ & Female & C-section & 3480 & & 3,6 \\
\hline Infant $2 \mathrm{CS}$ & Female & C-section & 3340 & & 3,5 \\
\hline Infant $3 \mathrm{CS}$ & Male & C-section & 3515 & & 3,12 \\
\hline Infant $4 \mathrm{CS}$ & Male & C-section & 4160 & $22 \mathrm{mo} \mathrm{PcV}$. & 3,6 \\
\hline Infant $5 \mathrm{CS}$ & Male & C-section & 4240 & 2 mo PcV, 3 mo Amp, 13 mo PcV, Amp. & 3,12 \\
\hline Infant $6 \mathrm{CS}$ & Male & C-section & 2800 & & 3,8 \\
\hline Infant $7 \mathrm{CS}$ & Female & C-section & 3690 & & 3,12 \\
\hline Infant $8 \mathrm{CS}$ & Male & C-section & 4785 & & 3,24 \\
\hline Infant $9 \mathrm{CS}$ & Male & C-section & 4775 & & 3,18 \\
\hline
\end{tabular}


Supplementary Table 3. Mean relative abundance (\%) and standard deviation (SD) of the most abundant phyla found at different time points in the infants born by vaginal delivery $(15 ; \mathrm{VD})$ and caesarean section $(9 ; \mathrm{CS})$ and in the mothers. Statistical analysis of phylum abundances between the VD and CS infants at the various time points and between the mothers was performed using Wilcoxon rank-sum test. To correct for multiple testing, the P-values were converted to False Discovery Rate values (Q-values).

\begin{tabular}{|c|c|c|c|c|c|c|}
\hline Time & Delivery mode & $\begin{array}{c}\text { Actinobacteria } \\
\text { Mean \% (SD) }\end{array}$ & $\begin{array}{l}\text { Bacteroidetes } \\
\text { Mean \% (SD) }\end{array}$ & $\begin{array}{l}\text { Firmicutes } \\
\text { Mean \% (SD) }\end{array}$ & $\begin{array}{c}\text { Proteobacteria } \\
\text { Mean \% (SD) }\end{array}$ & $\begin{array}{c}\text { Verrucomicrobia } \\
\text { Mean \% (SD) }\end{array}$ \\
\hline \multirow[t]{2}{*}{1 week } & VD & $12(16)$ & $* 27(28)$ & $44(32)$ & $16(18)$ & $0(0)$ \\
\hline & $\mathrm{CS}$ & $11(19)$ & $* 3(8)$ & $57(21)$ & $29(16)$ & $0(0)$ \\
\hline \multirow[t]{2}{*}{1 month } & VD & 37 (29) & $21(25)$ & $32(23)$ & $10(8)$ & $0(0)$ \\
\hline & $\mathrm{CS}$ & $29(23)$ & $8(15)$ & $44(22)$ & $19(15)$ & $0(0)$ \\
\hline \multirow[t]{2}{*}{3 months } & VD & $45(31)$ & $* * \wedge 19(29)$ & $29(25)$ & $* 7(8)$ & $0(0)$ \\
\hline & $\mathrm{CS}$ & $48(30)$ & $* * \wedge 4(10)$ & $33(28)$ & *15 (14) & $0(0)$ \\
\hline \multirow[t]{2}{*}{6 months } & VD & $33(24)$ & $11(16)$ & $48(23)$ & $7(9)$ & $0(0)$ \\
\hline & $\mathrm{CS}$ & $36(33)$ & $3(6)$ & $54(30)$ & $7(5)$ & $0(0)$ \\
\hline \multirow[t]{2}{*}{12 months } & VD & $12(11)$ & $* 14(11)$ & $69(15)$ & $3(6)$ & $1(1)$ \\
\hline & $\mathrm{CS}$ & $15(14)$ & *6 (12) & $69(16)$ & $5(7)$ & $5(9)$ \\
\hline \multirow[t]{2}{*}{24 months } & VD & $7(4)$ & $13(7)$ & $77(7)$ & $0(0)$ & $2(3)$ \\
\hline & $\mathrm{CS}$ & $10(9)$ & $11(8)$ & $77(11)$ & $1(1)$ & $1(2)$ \\
\hline \multirow[t]{2}{*}{ Mothers } & VD & $7(7)$ & $16(11)$ & $74(21)$ & $3(7)$ & $1(1)$ \\
\hline & $\mathrm{CS}$ & $12(15)$ & $13(10)$ & $71(17)$ & $2(5)$ & $2(4)$ \\
\hline
\end{tabular}

$*=\mathrm{P}<0.05, * *=\mathrm{P}<0.01, \wedge=\mathrm{Q}<0.05$. 
Supplementary Table 4. Mean relative abundance (\%) and standard deviation (SD) of the most abundant genera found at different time points in the infants born by vaginal delivery $(15 ; \mathrm{VD})$ and caesarean section $(9 ; \mathrm{CS})$ and in the mothers. Only genera displaying $>1 \%$ average abundance were included. Statistical analysis of genera abundances between the VD and CS infants at the various time points and between the mothers was performed using Wilcoxon rank-sum test. To correct for multiple testing, the P-values were converted to False Discovery Rate values (Q-values).

\begin{tabular}{|c|c|c|c|c|c|c|c|}
\hline Vaginal delivery & $\begin{array}{c}1 \text { week } \\
\text { Mean \%(SD) } \\
\mathbf{n}=15 \\
\end{array}$ & $\begin{array}{c}1 \text { month } \\
\text { Mean \%(SD) } \\
n=15 \\
\end{array}$ & $\begin{array}{c}3 \text { months } \\
\text { Mean \%(SD) } \\
n=15 \\
\end{array}$ & $\begin{array}{c}6 \text { months } \\
\text { Mean \%(SD) } \\
n=15 \\
\end{array}$ & $\begin{array}{c}12 \text { months } \\
\text { Mean \%(SD) } \\
n=15 \\
\end{array}$ & $\begin{array}{c}24 \text { months } \\
\text { Mean \%(SD) } \\
\text { n=15 } \\
\end{array}$ & $\begin{array}{c}\text { Mothers } \\
\text { Mean \%(SD) } \\
\text { n=15 } \\
\end{array}$ \\
\hline Bifidobacterium & $11.6(16.2)$ & $34.4(30)$ & $44.7(32.2)$ & $32.1(23.7)$ & $10.5(10.6)$ & $4.9(3.3)$ & $5.3(7)$ \\
\hline Bacteroides & *24.1(26.3) & $17.3(25.2)$ & *14.6(28.6) & $10.2(16.1)$ & $10(9.3)$ & $7.4(6.2)$ & $8.2(9.2)$ \\
\hline Parabacteroides & $2.8(6.6)$ & $* 3.3(5.3)$ & $2.5(7.2)$ & $0.7(1.5)$ & $* 0.9(2.5)$ & $1(2.4)$ & $0.8(1)$ \\
\hline Staphylococcus & $6.9(7.4)$ & $1.3(1.7)$ & $0.2(0.4)$ & $0.1(0.5)$ & $0(0)$ & $0(0)$ & $0.1(0.4)$ \\
\hline Enterococcus & $3.3(9.4)$ & $* * \wedge 0(0.1)$ & $1.8(3.8)$ & $1.2(3.1)$ & $4.5(16.5)$ & $0(0.1)$ & $0.1(0.2)$ \\
\hline Lactobacillus & $1(2.1)$ & $3.2(5.7)$ & $3.6(7)$ & $2.4(6.5)$ & $* 0(0)$ & $0(0.2)$ & $0.3(0.6)$ \\
\hline Streptococcus & $15.8(17.2)$ & $7.8(7.2)$ & $4.1(9.8)$ & $4.2(4.6)$ & $6.5(10.4)$ & $3.7(4.9)$ & $1.4(1.6)$ \\
\hline Lachnospira & $0.4(1.1)$ & $0.4(1.2)$ & $0.9(1.6)$ & $1.3(1.5)$ & $3.3(2.2)$ & $4.9(2.2)$ & $6.1(2.6)$ \\
\hline Lachnospiraceae Incertae Sedis & $0.4(1.1)$ & $0.4(1.2)$ & $0.9(1.6)$ & $1.3(1.5)$ & $3.3(2.2)$ & $4.9(2.2)$ & $6.1(2.6)$ \\
\hline Unclassified_Lachnospiraceae & $0.3(1.2)$ & $0(0.1)$ & $0.4(1.6)$ & $1.7(2.8)$ & $7.3(6.5)$ & $10.9(6.3)$ & $5.9(3.2)$ \\
\hline Peptostreptococcaceae Incertae Sedis & $1.4(4.1)$ & $0.4(1)$ & $0.7(1.2)$ & $3.6(6.9)$ & $3.1(4)$ & $3(3)$ & $4.5(5.2)$ \\
\hline Faecalibacterium & $0.1(0.4)$ & $0(0)$ & $0(0.1)$ & $1.7(2.8)$ & $3.1(3.5)$ & $4.6(4)$ & $3.5(3)$ \\
\hline Ruminococcus & $0.3(1)$ & $0(0)$ & $0(0.1)$ & $0.2(0.5)$ & $3.4(4)$ & $4.2(3.5)$ & $6.1(5.2)$ \\
\hline Unclassified_Ruminococcaceae & $0.3(0.8)$ & $0(0.1)$ & $0.4(1.2)$ & $0.4(0.8)$ & $* 1(1.3)$ & $2.1(1.6)$ & $4.6(3.7)$ \\
\hline Veillonella & $* 3.3(4.3)$ & $1.5(1.6)$ & $2.4(3.3)$ & $6.2(5.8)$ & $* * 0.4(0.8)$ & $0.1(0.2)$ & $0(0.1)$ \\
\hline Erysipelotrichaceae Incertae Sedis & $1.1(2.7)$ & $4.6(10.9)$ & $2.5(5.5)$ & $1.7(4.1)$ & $2.2(2.5)$ & $1.5(2.2)$ & $0.9(1.1)$ \\
\hline Unclassified_Enterobacteriaceae & $13.1(19.3)$ & $4(7)$ & $5(7.9)$ & $3.6(9)$ & $* 1.4(4.4)$ & $0.2(0.3)$ & $2(7.6)$ \\
\hline
\end{tabular}

\begin{tabular}{|c|c|c|c|c|c|c|c|}
\hline Caesarean section delivery & $\begin{array}{c}1 \text { week } \\
\text { Mean \%(SD) } \\
\text { n=9 } \\
\end{array}$ & $\begin{array}{c}1 \text { month } \\
\text { Mean \%(SD) } \\
n=9 \\
\end{array}$ & $\begin{array}{c}3 \text { months } \\
\text { Mean \%(SD) } \\
n=9\end{array}$ & $\begin{array}{c}6 \text { months } \\
\begin{array}{c}\text { Mean \%(SD) } \\
n=9\end{array} \\
\end{array}$ & $\begin{array}{c}12 \text { months } \\
\text { Mean \%(SD) } \\
\text { n=9 }\end{array}$ & $\begin{array}{c}24 \text { months } \\
\text { Mean \%(SD) } \\
\text { n=9 }\end{array}$ & $\begin{array}{c}\text { Mothers } \\
\text { Mean \%(SD) } \\
\mathbf{n}=9 \\
\end{array}$ \\
\hline Bifidobacterium & $11(20.5)$ & $27.8(24.5)$ & $47.8(31.4)$ & $34.8(33.6)$ & $14.2(14.6)$ & $8.5(9)$ & $10.4(16.8)$ \\
\hline Bacteroides & $* 2.8(8.4)$ & $4.1(12.1)$ & $* 3.6(10.9)$ & $3.1(5.8)$ & $5.1(11.3)$ & $7.9(7.1)$ & $6.4(5.5)$ \\
\hline Parabacteroides & $0(0)$ & $* 0(0)$ & $0(0)$ & $0(0)$ & $* 0.1(0.3)$ & $0.4(0.4)$ & $0.7(0.9)$ \\
\hline Staphylococcus & $5.8(7.9)$ & $1.4(1.7)$ & $0.1(0.2)$ & $0(0)$ & $0(0)$ & $0(0)$ & $0(0)$ \\
\hline Enterococcus & $15.7(20)$ & **^7.2(9.5) & $3.1(3.6)$ & $2(2.8)$ & $1.2(2.2)$ & $0(0)$ & $0(0)$ \\
\hline Lactobacillus & $0(0)$ & $0.5(1.5)$ & $4.9(3.6)$ & $4.8(8.6)$ & $* 1.6(4.4)$ & $0(0)$ & $0.8(1.5)$ \\
\hline Streptococcus & $14.6(17.6)$ & $4.9(4.1)$ & $1.5(1)$ & $2.2(2.1)$ & $2.5(3.1)$ & $3.2(5.5)$ & $3.7(6)$ \\
\hline Lachnospira & $0(0)$ & $4.2(8.5)$ & $0(0.1)$ & $1.8(2)$ & $2.3(2.7)$ & $4.9(2.6)$ & $4.4(2.4)$ \\
\hline Lachnospiraceae Incertae Sedis & $0(0)$ & $4.2(8.5)$ & $0(0.1)$ & $1.8(2)$ & $2.3(2.7)$ & $4.9(2.6)$ & $4.4(2.4)$ \\
\hline Unclassified_Lachnospiraceae & $0(0)$ & $0(0)$ & $0.1(0.2)$ & $3.1(6.2)$ & $3.4(5.2)$ & $10.6(4.9)$ & $6.2(4.8)$ \\
\hline Peptostreptococcaceae Incertae Sedis & $0.2(0.6)$ & $0.6(1.6)$ & $0.4(0.9)$ & $2.2(2.7)$ & $4.5(5.2)$ & $3(4.2)$ & $1.4(1.6)$ \\
\hline Faecalibacterium & $0(0)$ & $0(0)$ & $0(0)$ & $0.2(0.6)$ & $1.4(2.6)$ & $3.5(2.7)$ & $2(1.8)$ \\
\hline Ruminococcus & $0(0)$ & $0(0)$ & $0(0)$ & $0(0)$ & $1.6(2.7)$ & $4.7(2.6)$ & $5.7(3.5)$ \\
\hline Unclassified_Ruminococcaceae & $0(0)$ & $0.2(0.5)$ & $0(0)$ & $0.1(0.1)$ & $* 0.3(0.7)$ & $1.4(1.7)$ & $3.4(3)$ \\
\hline Veillonella & $* 11.2(8.5)$ & $3.4(7.4)$ & $3.1(3.9)$ & $3.2(2.4)$ & $* * 2.4(2.3)$ & $0.3(0.3)$ & $0.1(0.3)$ \\
\hline Erysipelotrichaceae Incertae Sedis & $0.1(0.2)$ & $0.6(1.3)$ & $0.2(0.7)$ & $2.3(3.4)$ & $6.2(7.5)$ & $1.5(1.9)$ & $1.2(2.7)$ \\
\hline Unclassified_Enterobacteriaceae & $6.2(14.4)$ & $7(14.6)$ & $7.6(8.1)$ & $5.2(5.7)$ & $* 3.4(5.3)$ & $0.4(0.7)$ & $1.5(4.3)$ \\
\hline
\end{tabular}

\footnotetext{
$*=\mathrm{P}<0.05, * *=\mathrm{P}<0.01, \wedge=\mathrm{Q}<0.05$.
} 
Supplementary Table 5. Repeated measures ANOVAs to test whether there were any significant differences in Shannon diversity between delivery mode (VD and CS) when including the diversity indices from all time points (one week, one, three, six, twelve and 24 months). The ANOVAs where calculated on non-log Shannon diversity indicies from 15 vaginal delivered and nine caesarean section delivered infants, except for a few time points where the data point was missing due to subsampling (14 VD and 9 CS at one week; 15 VD and 8 CS at one month, 14 VD and 9 CS at twelve months).

\begin{tabular}{|l|c|}
\hline Taxa & P-value \\
\hline Total microbiota & $\mathbf{0 . 0 4 7}$ \\
\hline Firmicutes & 0.062 \\
\hline Proteobacteria & 0.903 \\
\hline Actinobacteria & 0.132 \\
\hline Bacteroidetes & $\mathbf{0 . 0 0 2}$ \\
\hline
\end{tabular}


Supplementary Table 6. The Shannon diversity index of the total microbiota and most abundant phyla in stool samples obtained at various ages from vaginal (VD) or caesarean section (CS) delivered infants as well as from the mothers.

\begin{tabular}{|c|c|c|c|c|c|}
\hline D & $\begin{array}{l}\text { Vaginal } \\
\text { delivery }\end{array}$ & & $\begin{array}{c}\text { Caesarean } \\
\text { section }\end{array}$ & & P-value* \\
\hline & median & iqr** & median & iqr** & \\
\hline 1 week & $\mathrm{n}=14 \#$ & & $\mathrm{n}=\mathbf{9}$ & & \\
\hline Total microbiota & 1.66 & $1.49-1.83$ & 1.46 & $1.38-1.61$ & 0.096 \\
\hline Firmicutes & 1.49 & $1.20-1.70$ & 1.18 & $0.78-1.49$ & 0.072 \\
\hline Proteobacteria & 0.09 & $0.00-0.22$ & 0.12 & $0.07-0.38$ & 0.393 \\
\hline Actinobacteria & 0.02 & $0.00-0.29$ & 0.05 & $0.00-0.52$ & 0.511 \\
\hline Bacteroidetes & 0.30 & $0.00-1.11$ & 0.00 & $0.00-0.03$ & 0.077 \\
\hline 1 month & $n=15$ & & $\mathbf{n}=\mathbf{8} \#$ & & \\
\hline Total microbiota & 1.59 & $1.50-1.97$ & 1.57 & $1.45-1.67$ & 0.681 \\
\hline Firmicutes & 1.27 & $1.02-1.68$ & 1.31 & $0.97-1.68$ & 0.925 \\
\hline Proteobacteria & 0.16 & $0.00-0.44$ & 0.00 & $0.00-0.04$ & 0.058 \\
\hline Actinobacteria & 0.13 & $0.03-0.75$ & 0.23 & $0.05-1.01$ & 0.457 \\
\hline Bacteroidetes & 0.42 & $0.00-0.81$ & 0.00 & $0.00-0.00$ & 0.022 \\
\hline 3 months & $n=15$ & & $\mathrm{n}=\mathbf{9}$ & & \\
\hline Total microbiota & 1.73 & $1.09-2.01$ & 1.45 & $1.21-1.77$ & 0.369 \\
\hline Firmicutes & 1.43 & $1.16-1.71$ & 1.45 & $1.03-1.77$ & 0.975 \\
\hline Proteobacteria & 0.19 & $0.07-0.45$ & 0.26 & $0.17-0.41$ & 0.395 \\
\hline Actinobacteria & 0.45 & $0.05-0.67$ & 0.14 & $0.05-0.61$ & 0.682 \\
\hline Bacteroidetes & 0.64 & $0.00-0.87$ & 0.00 & $0.00-0.00$ & 0.004 \\
\hline 6 months & $n=15$ & & $\mathbf{n}=\mathbf{9}$ & & \\
\hline Total microbiota & 2.48 & $1.55-2.68$ & 2.06 & $1.53-2.52$ & 0.29 \\
\hline Firmicutes & 2.25 & $1.65-2.44$ & 1.81 & $1.66-2.30$ & 0.482 \\
\hline Proteobacteria & 0.67 & $0.23-0.78$ & 0.27 & $0.09-0.59$ & 0.238 \\
\hline Actinobacteria & 0.51 & $0.38-0.63$ & 0.64 & $0.09-0.74$ & 0.976 \\
\hline Bacteroidetes & 0.62 & $0.00-1.09$ & 0.09 & $0.00-0.22$ & 0.202 \\
\hline 12 months & $\mathrm{n}=14 \#$ & & $\mathbf{n}=\mathbf{9}$ & & \\
\hline Total microbiota & 3.15 & $2.67-3.44$ & 2.28 & $2.18-2.82$ & 0.013 \\
\hline Firmicutes & 2.90 & $2.09-3.16$ & 2.04 & $1.91-2.86$ & 0.046 \\
\hline Proteobacteria & 0.70 & $0.00-0.96$ & 0.61 & $0.23-0.77$ & 0.506 \\
\hline Actinobacteria & 0.51 & $0.25-0.75$ & 0.59 & $0.11-0.72$ & 0.801 \\
\hline Bacteroidetes & 1.23 & $0.50-1.82$ & 0.00 & $0.00-0.74$ & 0.023 \\
\hline 24 months & $n=15$ & & $\mathbf{n}=\mathbf{9}$ & & \\
\hline Total microbiota & 3.33 & $3.23-3.71$ & 3.23 & $2.93-3.46$ & 0.194 \\
\hline Firmicutes & 3.10 & $2.91-3.36$ & 3.19 & $2.64-3.36$ & 0.682 \\
\hline Proteobacteria & 0.50 & $0.34-0.61$ & 0.13 & $0.00-0.39$ & 0.035 \\
\hline Actinobacteria & 0.72 & $0.48-1.23$ & 0.49 & $0.29-0.82$ & 0.123 \\
\hline
\end{tabular}




$\begin{array}{lccccc}\text { Bacteroidetes } & 1.83 & 1.19-2.21 & 1.03 & 0.49-1.37 & \mathbf{0 . 0 1 8} \\ \text { Mothers } & \mathbf{n = 1 5} & & \mathbf{n = 9} & & \\ \text { Total microbiota } & 3.99 & 1.35-3.79 & 3.78 & 3.45-3.97 & 0.084 \\ \text { Firmicutes } & 3.65 & 3.48-3.88 & 3.38 & 3.21-3.68 & 0.073 \\ \text { Proteobacteria } & 0.63 & 0.07-1.08 & 0.35 & 0.12-0.87 & 0.309 \\ \text { Actinobacteria } & 0.86 & 0.62-1.35 & 1.0 & 0.64-1.21 & 0.64 \\ \text { Bacteroidetes } & 1.97 & 1.31-2.45 & 2.20 & 1.63-2.37 & 0.907 \\ & & & & & \\ \text { *Wilcoxon rank-sum test. } \\ \text { **interquartile range. } \\ \text { \# Missing data points due to the subsampling of the data and exclusion of samples } \\ \text { With less than 1400 reads per sample. }\end{array}$


Supplementary Table 7. Statistical testing of mother-child overlap in microbial community composition. For each time point and for each infant we calculated the number of sequence types shared with its mother/number of sequence types observed in the infant $\left(\mathrm{R}_{\text {own }}\right)$. Likewise we calculated the average number of sequence types shared with other mothers/number of sequence types observed in the infant $\left(\mathrm{R}_{\mathrm{other}}\right)$. We then compared the $R_{\text {own }}$ and $R_{\text {other }}$ values pairwise for all infants within each group (VD or CS) with the Wilcoxon signed rank test. P-values for these tests are reported below, considering either sequences belonging to specific phyla or all sequences.

\begin{tabular}{lclllll}
\hline & \multicolumn{5}{c}{ Vaginal delivery } \\
\hline & 1 week & 1 month & 3 months & 6 months & 12 months & 24 months \\
Firmicutes & 0.421 & 0.804 & 0.191 & $\mathbf{0 . 0 4 1}$ & 0.073 & $\mathbf{0 . 0 4 8}$ \\
Proteobacteria & 0.724 & 1 & 0.932 & 0.088 & 0.49 & 0.834 \\
Actinobacteria & 0.308 & 0.53 & 0.551 & 0.916 & 0.802 & 0.187 \\
Bacteroidetes & 0.147 & $\mathbf{0 . 0 4 2}$ & 0.224 & $\mathbf{0 . 0 1 8}$ & 0.802 & 0.095 \\
All taxa & $\mathbf{0 . 0 3}$ & 0.121 & 0.074 & 0.064 & 0.055 & $\mathbf{0 . 0 2 2}$ \\
& & \multicolumn{7}{c}{ Caesarean section } \\
& & & & & \\
Firmicutes & 0.834 & 0.183 & 0.359 & 0.496 & 0.652 & 0.57 \\
Proteobacteria & 0.284 & 0.905 & 1 & 0.812 & 0.858 & 0.933 \\
Actinobacteria & 1 & 0.722 & 0.678 & 0.945 & 0.098 & 0.652 \\
Bacteroidetes & 0.461 & 0.371 & 1 & 0.786 & 0.352 & 1 \\
All taxa & 0.813 & 0.301 & 0.363 & 0.359 & 0.652 & 0.734 \\
\hline
\end{tabular}

